\title{
SORLA facilitates insulin receptor signaling in adipocytes and exacerbates obesity
}

\author{
Vanessa Schmidt, ${ }^{1}$ Nadja Schulz, ${ }^{2,3}$ Xin Yan, ${ }^{1}$ Annette Schürmann, ${ }^{2,3}$ Stefan Kempa, ${ }^{1}$ Matthias Kern, ${ }^{4}$ Matthias Blüher, ${ }^{4}$ \\ Matthew N. Poy, ${ }^{1}$ Gunilla Olivecrona, ${ }^{5}$ and Thomas E. Willnow' \\ 'Max-Delbrueck-Center for Molecular Medicine, Berlin, Cermany. ${ }^{2}$ Cerman Institute of Human Nutrition, Potsdam-Rehbruecke, Nuthetal, Germany. ${ }^{3}$ Cerman Center for Diabetes Research, \\ Munich-Neuherberg, Germany. ${ }^{4}$ University Medical Center, University of Leipzig, Leipzig, Germany. ${ }^{5}$ Department of Medical Biosciences and Physiological Chemistry, Umeå University, Umeå, Sweden.
}

\begin{abstract}
In humans, genetic variation of sortilin-related receptor, L(DLR class) A repeats containing (SORL1), which encodes the intracellular sorting receptor SORLA, is a major genetic risk factor for familial and sporadic forms of Alzheimer's disease. Recent CWAS analysis has also associated SORL1 with obesity in humans and in mouse models, suggesting that this receptor may play a role in regulating metabolism. Here, using mouse models with genetic loss or tissue-specific overexpression of SORLA as well as data from obese human subjects, we observed a gene-dosage effect that links SORLA expression to obesity and glucose tolerance. Overexpression of human SORLA in murine adipose tissue blocked hydrolysis of triacylglycerides and caused excessive adiposity. In contrast, Sorl1 gene inactivation in mice accelerated breakdown of triacylglycerides in adipocytes and protected animals from diet-induced obesity. We then identified the underlying molecular mechanism whereby SORLA promotes insulin-induced suppression of lipolysis in adipocytes. Specifically, we determined that SORLA acts as a sorting factor for the insulin receptor (IR) that redirects internalized receptor molecules from endosomes to the plasma membrane, thereby enhancing IR surface expression and strengthening insulin signal reception in target cells. Our findings provide a molecular mechanism for the association of SORL1 with human obesity and confirm a genetic link between neurodegeneration and metabolism that converges on the receptor SORLA.
\end{abstract}

\section{Introduction}

Obesity is a global health problem that poses a major risk factor for life-threatening diseases such as cardiovascular disease, cancer, and neurodegeneration. Recent GWAS have identified loci associated with obesity in humans, paving the way for potential therapeutic strategies to combat metabolic disorders (1-4). However, the molecular mechanisms of many novel loci associated with metabolic traits remain poorly understood, mandating functional studies to elucidate their mode of action. One such example is $S O R L 1$, the gene encoding sorting-related receptor with type A repeats (SORLA).

SORLA is a member of the VPS10P domain receptor gene family, a group of type 1 transmembrane receptors that traffic target proteins among the Golgi apparatus, cell surface, and endosomes in various mammalian cell types (reviewed in ref. 5). SORLA is best known for its function as a sorting protein for the amyloid precursor protein (APP), the main etiologic agent in Alzheimer's disease (AD). SORLA-mediated sorting of APP prevents proteolytic breakdown of APP into neurotoxic amyloid- $\beta$ peptides, protecting the brain from neurodegenerative processes $(6,7)$. The genome-wide association of SORL1 gene variants with sporadic AD confirms the relevance of SORLA for neurodegenerative disease in the human population (8). Surprisingly, recent GWAS have also associated SORL1 with several metabolic traits (e.g., obesity, waist circumference) in humans and in mouse mod-

Conflict of interest: The authors have declared that no conflict of interest exists. Submitted: September 15, 2015; Accepted: April 29, 2016.

Reference information: J Clin Invest. 2016;126(7):2706-2720. doi:10.1172/JCI84708. els, suggesting a function for this receptor in metabolic regulation $(2,9)$. How exactly SORLA affects energy homeostasis and the occurrence of obesity remains to be clarified.

Studying mouse models with loss of function or tissue-specific overexpression of SORLA as well as obese human subjects, we identified a role for this receptor in the control of lipid turnover in adipose tissue. Specifically, SORLA facilitates functional expression of the insulin receptor (IR) to promote insulin-dependent suppression of lipolysis in adipocytes. Overexpression of SORLA in adipose tissue blocks hydrolysis of triacylglycerides to free fatty acids (FFAs) and enhances fat deposition. In contrast, Sorl1 gene inactivation accelerates FFA release from triacylglycerides and protects mice from diet-induced obesity.

\section{Results}

Loss of SORLA reduces white adipose tissue deposition in mice. GWAS identified single nucleotide polymorphisms in chromosomal loci in the proximity of SORL1 as being associated with waist circumference in humans (2) and with body fat percentage in mice (9). To substantiate SORL1 as the gene responsible for these metabolic traits, we analyzed weight and body composition of Sorl1mice that are genetically deficient for this receptor (referred to as SORLA KO) (6). When placed on a high-fat diet (HFD), SORLA $\mathrm{KO}$ animals showed a reduced gain in body weight compared with Sorl1 ${ }^{+/+}$littermate controls that express the receptor (referred to as SORLA WT) (Figure 1A). Although no obvious difference in body weight was seen on normal chow (Figure 1B), an inherent defect in acquisition of body fat was already apparent in normal chow-fed SORLA KO animals after their body composition was evaluated 

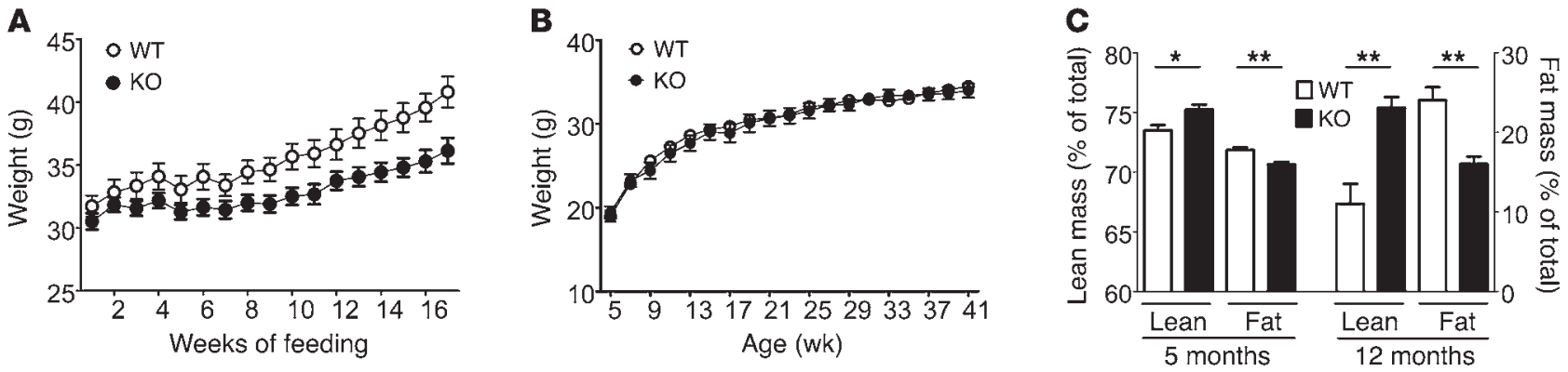

Figure 1. Loss of SORLA protects from diet-induced obesity. (A) Gain of body weight of SORLA WT and SORLA KO mice placed on a HFD at 35 weeks of age ( $n=11-12$ animals per genotype; 2-way ANOVA; $P=0.02$ for genotypes). (B) Weight gain of SORLA WT and SORLA KO mice on normal chow. (C) Lean and fat tissue mass (\% of total body weight) in SORLA WT and SORLA KO mice on normal chow as determined by NMR at 5 and 12 months of age. SORLA KO mice show an increase in lean and a concomitant decrease in fat tissue mass compared with SORLA WT animals. $n=5-6$ animals per genotype. ${ }^{*} P<0.05 ;{ }^{*} P<0.01$, unpaired Student's $t$ test.

by NMR. At both at 5 and 12 months of age, chow-fed SORLA KO mice showed a decrease in fat and a concomitant increase in lean body mass compared with littermate controls (Figure 1C). This alteration in body composition was not due to changes in food intake, locomotor activity, or heat production, as these parameters were comparable in SORLA WT and SORLA KO mice (Supplemental Figure 1; supplemental material available online with this article; doi:10.1172/JCI84708DS1). Together, these findings suggested the presence of an inherent defect in the accumulation of fat tissue mass in SORLA KO animals even on a normal diet.

Earlier work documented the ability of SORLA to bind lipoprotein lipase (LpL), the enzyme responsible for hydrolysis of triacylglycerides in circulating lipoproteins (10). To explore potential changes in systemic lipoprotein metabolism in SORLA KO mice, we characterized the plasma lipid levels and lipoprotein profiles in mice on a normal chow diet. SORLA KO mice showed a modest increase in FFAs and a concomitant decrease in triacylglyceride levels compared with SORLA WT animals. While plasma cholesterol levels were also slightly elevated (Table 1), the lipoprotein profiles were indistinguishable when comparing both genotypes (Supplemental Figure 2, A and B). Also, no difference in LpL activity was detected in postheparin plasma or in white or brown adipose tissues in SORLA KO compared with SORLA WT animals (Supplemental Figure 2C).

Overexpression of SORLA in adipocytes promotes obesity in humans and mouse models. So far, our studies confirmed the relevance of SORLA for the determination of fat mass in mice, further substantiating the association of the Sorl1 locus with obesity-related traits in mouse models (9). SORLA is expressed in several tissues relevant for metabolic control, including hypothalamus, liver, and skeletal muscle as well as white and brown adipose tissues (Supplemental Figure 3A). To identify the cell type responsible for SORLA-dependent control of fat mass, we devised an experimental model of tissue-specific overexpression of the receptor in vivo. To do so, we used a mouse model carrying an inducible human SORL1 cDNA construct in the Rosa26 locus that we had generated previously ( $\operatorname{Sorl1}^{+/+} \operatorname{Rosa}^{26^{\text {TgSORL1WT }}}$ ) (11). We crossed this mouse line with the adiponectin-Cre strain $\left(\mathrm{Tg}^{\text {(Adipoq-Cre)1Evdr/J })}\right.$ directing Cre recombinase activity to adipocytes (Supplemental Figure 3B). In line with this strategy, Cremediated removal of the transcription stop site preceding the SORLA expression cassette in Rosa26 resulted in overexpression of human SORLA in adipose tissues, but not in hypothalamus, liver, or muscle in Sorl1 ${ }^{+/+}$Rosa26 $6^{\text {TgSORL1WT }} \mathrm{Tg}^{\text {(Adipoq-Cre)1Evdr/J }}$ animals (referred to as SORLA Tg) (Supplemental Figure 3C). SORLA Tg mice were compared with Sorl1 ${ }^{+/+} \mathrm{Rosa26}^{+/+} \mathrm{Tg}^{\text {(Adipoq- }}$ Cre)1Evdr/J littermate controls carrying the Cre transgene, but lacking the SORL1 cDNA construct in their germ line (referred to as SORLA WT/Cre). Similar to the subcellular pattern seen for the endogenous murine receptor, the pattern in human SORLA was largely confined to intracellular vesicles in adipocytes of SORLA Tg mice as shown by immunohistology (Supplemental Figure

\section{Table 1. Plasma lipid parameters in mice on normal chow}

\begin{tabular}{|c|c|c|c|c|c|}
\hline Lipid parameters & \multicolumn{2}{|c|}{ SORLA WT } & \multicolumn{2}{|c|}{ SORLA KO } & \\
\hline FFAs (nmol/ml) & $455.38 \pm 35.55$ & $(n=32)$ & $595.35 \pm 32.56$ & $(n=33)$ & $P<0.01$ \\
\hline Cholesterol ( $\mu \mathrm{g} / \mathrm{ml})$ & $156.30 \pm 6.26$ & $(n=23)$ & $187.46 \pm 6.50$ & $(n=21)$ & $P<0.01$ \\
\hline FFAs (nmol/ml) & $498.13 \pm 27.33$ & $(n=18)$ & $574.78 \pm 35.87$ & $(n=19)$ & NS \\
\hline Triacylglyceride (mg/dl) & $104.22 \pm 8.07$ & $(n=18)$ & $103.34 \pm 11.06$ & $(n=19)$ & NS \\
\hline
\end{tabular}

Levels of the indicated lipids were determined using commercial ELISA kits in the plasma of male mice on normal chow (as detailed in Methods). Unpaired

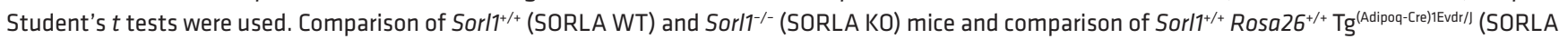
WT/Cre) and Sor/1+/+ Rosa26 TgSORLIWT $\mathrm{Tg}^{\text {(Adipoq-Cre)1Evdr/I }}$ (SORLA Tg) mice. 
A

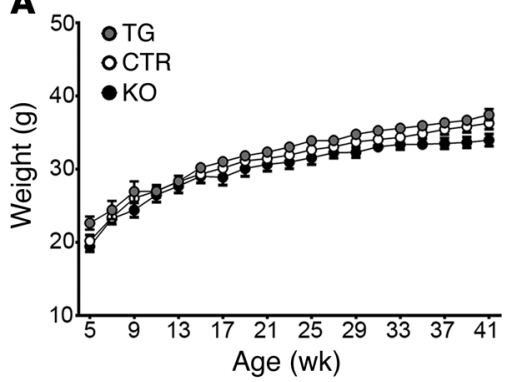

B

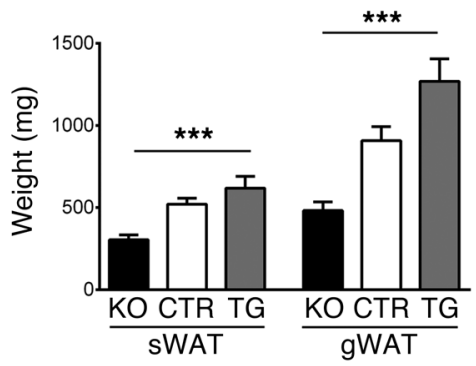

C
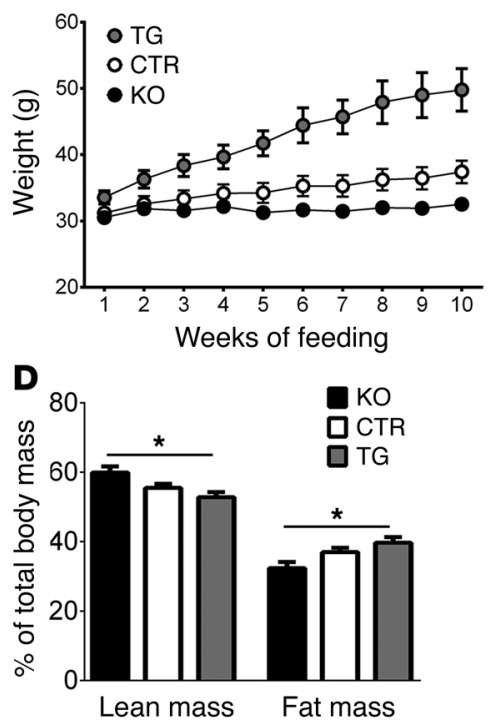

Figure 2. Overexpression of SORLA in adipose tissue exacerbates fat tissue acquisition. (A) Weight gain of mice of the indicated SORLA genotypes on normal chow. (B) Weight of subcutaneous WAT (sWAT) and gonadal WAT (gWAT) in mice of the indicated genotypes on normal chow. $n=6-7$ per genotype. ${ }^{* *} P<0.001,1$-way ANOVA. No statistically significant differences in parameters shown in $\mathbf{A}$ and $\mathbf{B}$ were noted when comparing SORLA WT and SORLA WT/ Cre mice ( $P>0.05$, unpaired Student's $t$ test). Thus, these 2 genotype groups were combined as the control group (CTR). (C) Gain in body weight of mice of the indicated genotypes placed on HFD at 20 week of age. $n=5-6$ animals per genotype. $P<0.001$ for genotype, 2 -way ANOVA. (D) Lean and fat tissue mass (\% of total body weight) as determined by NMR in mice fed with HFD for 15 weeks. SORLA levels positively correlate with an increase in fat and a concomitant decrease in lean tissue mass when comparing SORLA KO, SORLA CTR, and SORLA Tg animals. $n=9-10$ animals per genotype. ${ }^{*} P<0.05,1$-way ANOVA.
3D). This distribution is prototypical for SORLA and reflects its role in directing vesicular trafficking of target proteins between Golgi and endosomes (12).

Next, we performed comparative analyses of weight and body composition in SORLA Tg, SORLA WT/Cre, SORLA WT, and SORLA KO animals. As no obvious phenotypic differences were seen comparing Sorl1 ${ }^{+/+}$mice without (SORLA WT) or with the adiponectin-Cre transgene (SORLA WT/Cre) using unpaired Student's $t$ test, data obtained in both control lines were combined in a control strain data set (referred to as SORLA CTR). Similarly to what we observed for the comparison of SORLA WT and SORLA KO mice as shown in Figure 1 above, no difference in body weight was seen when comparing all genotypes on a normal chow diet (Figure 2A). Still, the genotype-dependent acquisition of fat tissue mass was already apparent, with the weights of subcutaneous and gonadal fat pads being highest in SORLA Tg and lowest in SORLA KO. SORLA CTR animals showed an intermediate phenotype (Figure 2B). No differences in plasma lipid levels (Table 1) or in lipoprotein profiles (Supplemental Figure 4) were seen when comparing SORLA Tg mice and SORLA WT/Cre littermates on normal chow. Also, SORLA Tg animals showed normal food intake, levels of activity, and heat production when compared with SORLA WT/Cre littermates (Supplemental Figure 5).

HFD feeding exacerbates obesity and causes glucose intolerance in mice overexpressing SORLA. The effect of SORLA in promoting fat tissue acquisition seen on normal chow (Figure 2B) was aggravated by HFD feeding of mice for 15 weeks. This dietary stress resulted in a higher increase in body weight gain in SORLA Tg animals (Figure 2C) and a more pronounced shift from lean to fat tissue mass (Figure 2D) as compared with SORLA KO animals. Again, SORLA CTR mice showed intermediate phenotypes (Figure 2, C and D). Plasma lipid levels were not significantly different comparing all genotypes (Table 2). However, histological analysis showed massive hypertrophy as well as extensive tissue remodeling in white adipose tissue (WAT) of SORLA Tg mice. Also, these animals exhibited excessive lipid accumulation in muscle and liver compared with SORLA WT/Cre littermates, supportive of overt obesity in mice overexpressing SORLA (Supplemental Figure 6A). Hypertrophy of adipocytes and lipid accumulation in muscle and liver were also observed in SORLA WT as compared with SORLA KO littermates (Supplemental Figure 6B).

The diet-induced obesity caused by overexpression of SORLA in adipose tissue resulted in increased fasting plasma glucose (Supplemental Figure 7A) and insulin (Supplemental Figure 7B) levels in SORLA Tg compared with SORLA WT/Cre control mice. Impaired glucose handling in SORLA Tg mice on a HFD was substantiated by decreased clearance of glucose during glucose tolerance test (Supplemental Figure 7, C and D). No differences in fasting plasma glucose and insulin levels (Supplemental Figure 7, A and B) or in glucose tolerance (Supplemental Figure 7, C and D) were seen when comparing SORLA Tg and SORLA WT/Cre animals on normal chow. In line with being protected from dietinduced obesity, SORLA KO animals on HFD showed significantly lower levels of fasting plasma glucose and insulin levels compared with their respective SORLA WT littermate controls (Supplemental Figure 7, E and F). No differences in fasting plasma glucose and insulin levels or in the glucose tolerance test were seen when comparing SORLA KO and SORLA WT mice on normal chow (Supplemental Figure 7, E-H).

SORLA levels correlate with BMI in humans. Thus far, our studies have identified a positive correlation of SORLA levels in adipocytes with the extent of fat tissue acquisition in mouse models. While this SORL1 gene dosage effect was already apparent on normal chow, the phenotype was further exacerbated by HFD feeding and resulted in overt obesity and secondary metabolic disturbances (e.g., glucose intolerance) in mice with high levels of receptor expression (SORLA Tg). Because all metabolic phenotypes seen in SORLA KO mice were contrasted by conditional overexpression of SORLA in adipose tissue in SORLA Tg animals, we reasoned that a primary role of the receptor in control of adipocyte metabolism is to underlie its impact on obesity. The rel- 

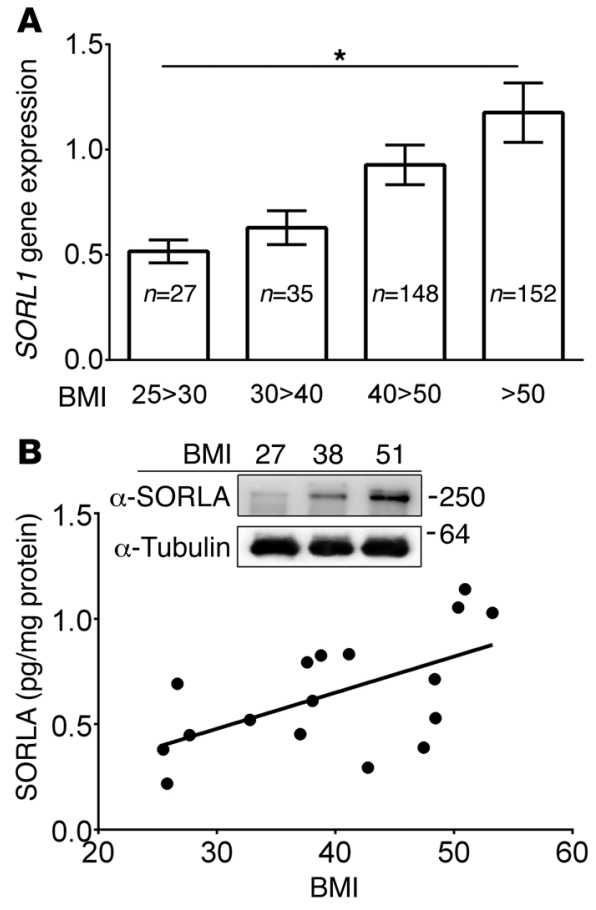

evance of SORLA expression levels in adipocytes for control of body weight in humans was further substantiated by studies in obese human subjects, documenting a clear correlation of BMI with levels of SORL1 transcript (Figure 3A) and SORLA protein (Figure 3B) in visceral adipose tissue.

SORLA levels determine lipolytic activity in WAT. To uncover the mechanisms of SORLA in controlling adipocyte metabolism, we studied the lipolytic activity in adipose tissue explants from SORLA KO and SORLA WT as well as from SORLA WT/Cre and SORLA Tg mice in detail. To exclude confounding secondary effects from dietary stress and pronounced obesity, we performed these analyses that readily document the SORLA-dependent effect on fat mass in tissues from mice on normal chow (Figure 1C and Figure 2B). As no obvious phenotypic differences were seen when comparing control littermates without (SORLA WT) or with the Cre transgene (SORLA WT/Cre) using unpaired Student's $t$ test $(P>0.05)$, data obtained in both control lines in all subsequent studies were combined in a control strain data set (SORLA CTR).
Figure 3. SORL1 transcript and protein levels in human WAT correlate with BMI. (A) Levels of SORL1 transcripts were determined by q-PCR in human visceral WAT and calculated as relative to the levels of 185 rRNA in the samples. SORL1 levels are positively correlated with BMI. ${ }^{*} P<0.05$, 1 -way ANOVA. (B) SORLA protein levels were determined in human visceral WAT by ELISA and positively correlated with BMI of sample donors using linear regression and correlation analysis $(P=0.013)$. The inset depicts Western blot analysis of SORLA levels in subcutaneous WAT of individuals with the indicated $\mathrm{BMI}$.

In adipose tissue explants, the amounts of intracellular and released FFAs were negatively correlated with SORLA activity, with the highest levels seen in SORLA KO, intermediate levels in SORLA CTR, and the lowest levels in SORLA Tg animals (Figure 4A). This finding suggested reduced lipolytic activity in adipocytes (over)expressing SORLA. This assumption was supported by the amount of released glycerol, the second product of lipolysis, that was also negatively correlated with SORLA levels comparing Sorl1 genotypes (Figure 4A). Lipidomics analyses identified a number of FFAs being increased in WAT of SORLA KO animals compared with the other genotypes (Supplemental Figure 8A). The same fatty acids were also increased in the plasma of SORLA KO mice (Supplemental Figure 8B). Excessive FFAs in mice with lower SORLA levels were likely deposited in peripheral tissues, as suggested by increased FFA concentrations in muscle of SORLA KO $(0.468 \pm 0.02 \mathrm{nmol} / \mathrm{mg}$ tissue $)$ versus SORLA WT controls $(0.396$ $\pm 0.02 \mathrm{nmol} / \mathrm{mg}$ tissue, $P<0.05)$ or in SORLA WT/Cre $(0.514 \pm$ $0.03 \mathrm{nmol} / \mathrm{mg}$ tissue $)$ versus SORLA Tg $(0.404 \pm 0.02 \mathrm{nmol} / \mathrm{mg}$ tissue, $P<0.05)$ mice on a normal chow diet.

In adipocytes, 3 intracellular lipases act in a sequential fashion to break down triacylglycerides to FFAs and glycerol (reviewed in refs. 13, 14). Initial hydrolysis of triacylglycerides to diacylglycerides is performed by adipose triglyceride lipase (ATGL). Subsequently, hormone-sensitive lipase (HSL) catalyzes the conversion of diacylglycerides to monoacylglycerides, while monoacylglycerol lipase (MGL) converts monoacylglycerides to glycerol. Consistent with the differences in FFA and glycerol levels documented in Figure 4A, the lipolytic activity in WAT explants was highest in SORLA KO and lowest in SORLA Tg animals (Figure 4B). We also quantified esterase activity, an activity that is unique to HSL and not seen for ATGL and MGL. Esterase (HSL) activity in the explants showed the same inverse correlation with SORLA levels as the lipase activity (Figure 4C).

Table 2. Plasma lipid parameters in mice on HFD

Lipid parameters

FFAs (nmol/ml)

Triacylglyceride $(\mathrm{mg} / \mathrm{dl})$

Free cholesterol $(\mu \mathrm{g} / \mathrm{ml})$

Lipid parameters

FFAs (nmol/ml)

Triacylglyceride (mg/dl)

Free cholesterol $(\mu \mathrm{g} / \mathrm{ml})$
SORLA WT

$973.34 \pm 69.28$
$67.5 \pm 9.98$
$511.98 \pm 33.45$
SORLA WT/Cre

$731.98 \pm 102.90$

$58.58 \pm 2.74$

$353.47 \pm 26.90$

$\begin{array}{lc}(n=9) & 978.95 \pm 83.70 \\ (n=9) & 58.15 \pm 3.01 \\ (n=9) & 426.88 \pm 46.86\end{array}$

$(n=9)$

$(n=9)$

$(n=9)$

$65.09 \pm 5.21$

$475.15 \pm 34.92$
SORLA KO

$\begin{array}{ll}(n=10) & \text { NS } \\ (n=10) & \text { NS } \\ (n=10) & \text { NS }\end{array}$

SORLA Tg

$\begin{array}{cc}(n=9) & \text { NS } \\ (n=9) & \text { NS } \\ (n=9) & p<0.05\end{array}$

Levels of the indicated lipids were determined using commercial ELISA kits in plasma of male mice on a HFD for 15 weeks (as detailed in Methods).

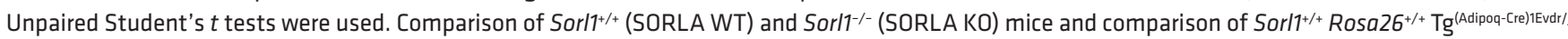

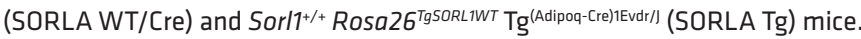



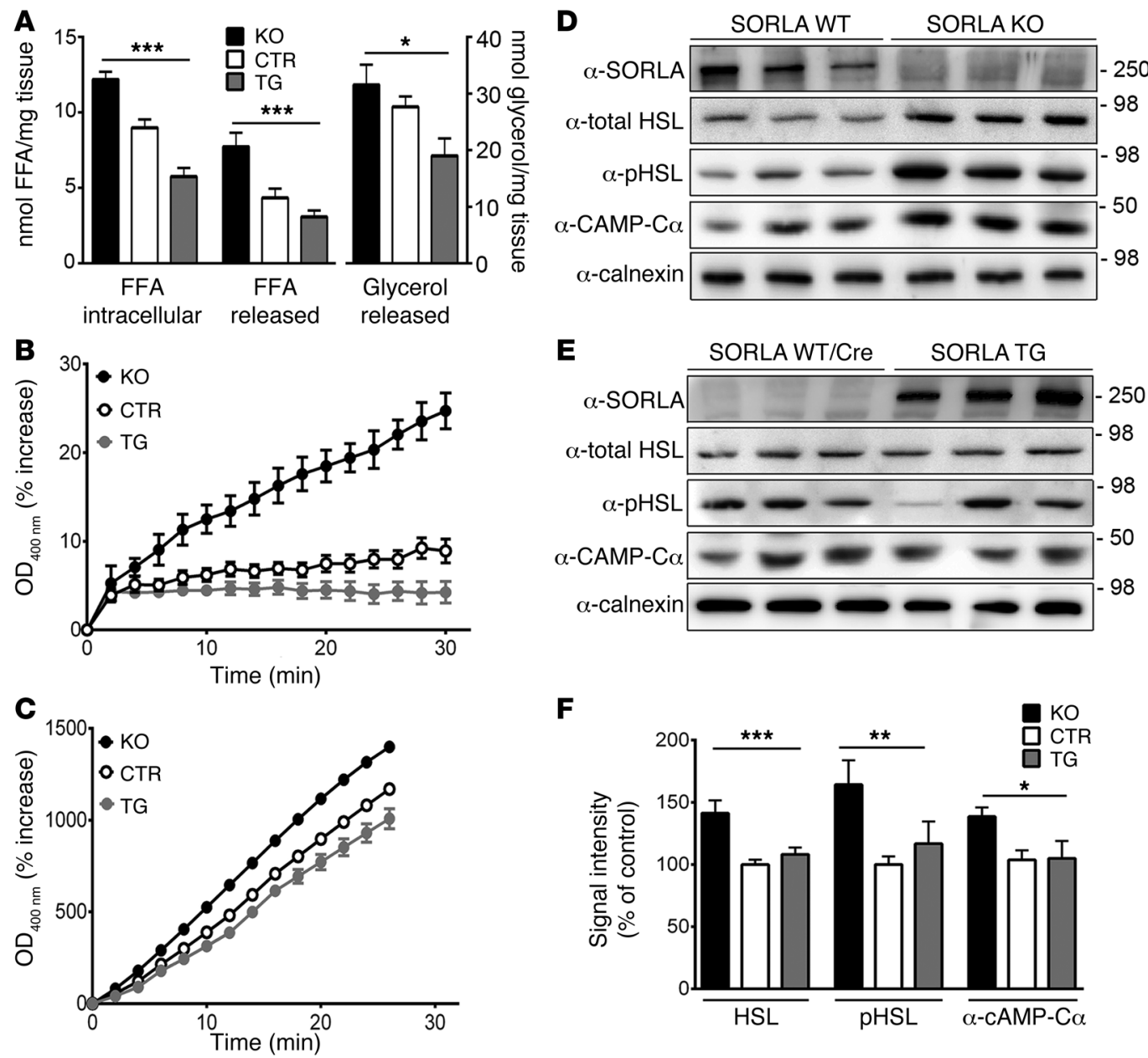

Figure 4. SORLA levels in WAT are inversely correlated with lipolytic enzyme activities. (A) Explants of gonadal WAT were generated from mice of the indicated genotypes and kept in culture for 72 hours. Levels of FFAs in the tissue (FFA intracellular) and the supernatant (FFA released) as well as levels of glycerol released negatively correlate with SORLA levels. $n=6-11$ animals per genotype; ${ }^{*} P<0.05 ;{ }^{* *} P<0.001,1$-way ANOVA. (B and C) Levels of lipase (B) and esterase activities (C) in adipocytes isolated from gonadal WAT were determined as described in Supplemental Methods and are expressed as a percentage of signal increase at $\mathrm{OD}_{400 n m}$ over time. Both enzyme activities are inversely correlated with SORLA levels, as seen in comparisons of SORLA KO, SORLA CTR, and SORLA Tg animals. $n=6-7$ animals per genotype. $P<0.001,2$-way ANOVA. Data in $\mathbf{A}-\mathbf{C}$ were determined separately in SORLA WT and SORLA WT/Cre animals. As no statistically significant difference in any parameter was noted, these 2 genotype groups were combined as the control group (CTR). $P>0.05$, unpaired Student's $t$ test. ( $D$ and $\mathbf{E}$ ) Levels of SORLA, total HSL and p-HSL, and cAMP-dependent protein kinase $A$ catalytic $\alpha$ subunit (CAMP-C $\alpha$ ) (activated PKA) as well as calnexin (loading control) were determined by Western blot analysis in gonadal WAT of SORLA WT and SORLA KO animals (D) or in SORLA WT/Cre and SORLA Tg animals (E). Representative samples from 3 animals per genotype are shown. (F) Signal intensities for the indicated proteins in SORLA KO and SORLA Tg were determined by densitometric scanning of replicate blots and expressed as relative value compared with the respective control group (SORLA WT or SORLA WT/Cre) set to $100 \%$. Both control groups are jointly referred to as CTR. A significant increase in HSL, p-HSL, and cAMP-C $\alpha$ levels in SORLA KO as compared with CTR and Tg mice was noted. $n=10-12$ animals per genotype. ${ }^{*} P<0.05 ;{ }^{* *} P<0.01 ;{ }^{* *} P<0.001,1$-way ANOVA.

HSL is the rate-limiting enzyme for regulated lipolysis in adipocytes. Its activity is controlled through phosphorylation by PKA, enabling the phosphorylated (activated) enzyme to access its substrate in lipid droplets $(15,16)$. In line with having the highest lipolytic activity, SORLA KO explants also contained significantly higher levels of total and of phosphorylated HSL (p-HSL) than SORLA CTR and Tg explants (Figure 4, D-F). Also, the amount of cAMP-dependent PKA catalytic $\alpha$ subunit (activated PKA) was highest in SORLA KO adipose tissue, as documented by Western blotting (Figure 4, D-F). The differences in HSL protein and activity were not explained by alterations in gene transcription, as $\mathrm{Hsl}$ transcript levels were lowest in SORLA KO, intermediate in SORLA CTR, and highest in SORLA Tg (Supplemental Figure 8C).
SORLA promotes insulin-dependent inhibition of lipolysis in adipocytes. The rate of lipolysis in adipose tissue is subject to tight regulation by several hormones (17). Chief factors are catecholamines (e.g., norepinephrine [NE]) that act through $\beta$-adrenergic receptors to stimulate cAMP production by adenylate cyclase (AC). In turn, cAMP activates PKA, resulting in increased HSL phosphorylation and activity. Stimulation of lipolysis in adipose tissue is counteracted by IR signaling that activates PKB (AKT). AKT promotes phosphodiesterase $3 \beta$ (PDE3 $\beta$ ) activity that converts cAMP to AMP and inhibits PKA action. To identify the signaling pathway affected by SORLA in adipocytes, we performed studies in gonadal fat explants from mice on normal chow. When explants from SORLA KO, SORLA WT, SORLA WT/Cre, and 
A

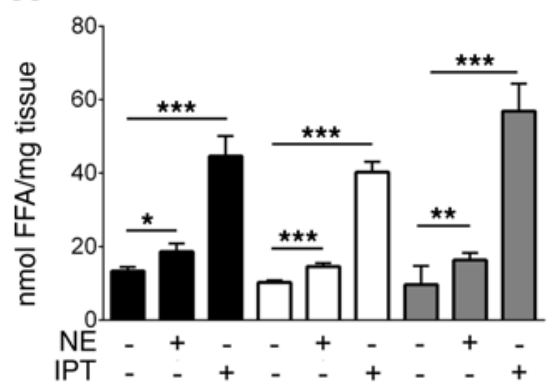

D

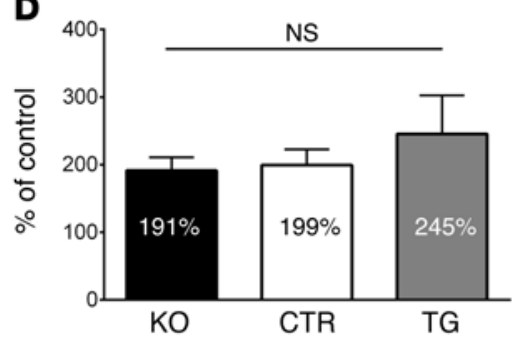

G
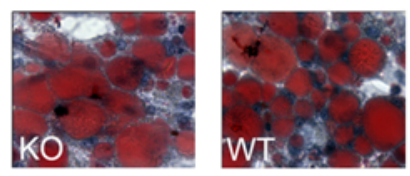

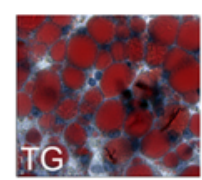

B

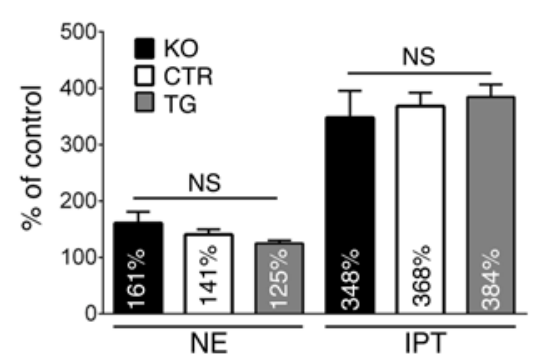

E

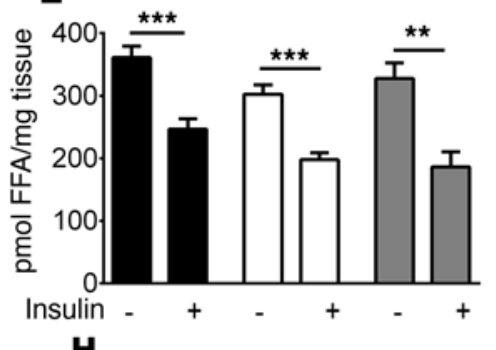

H

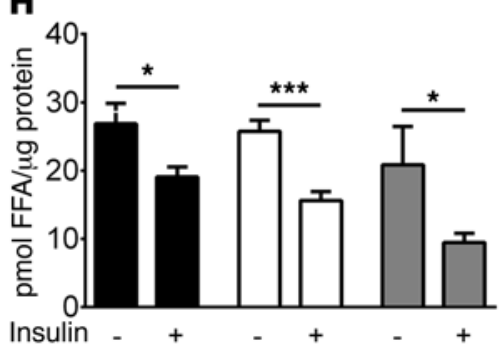

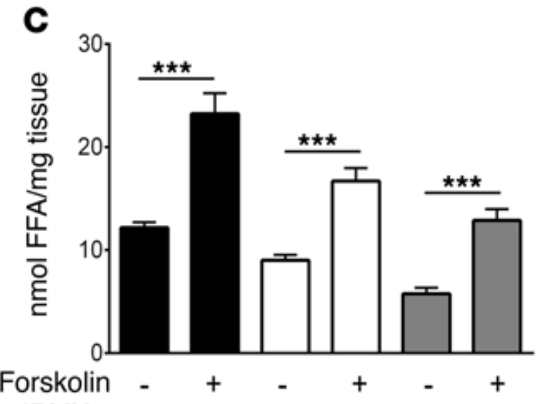

+ IBMX

F

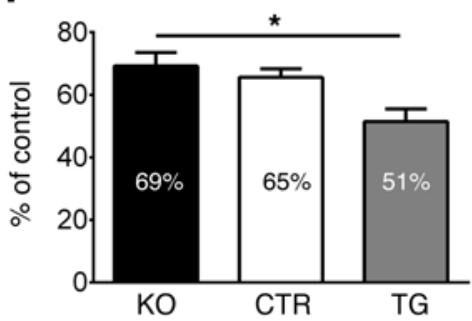

I

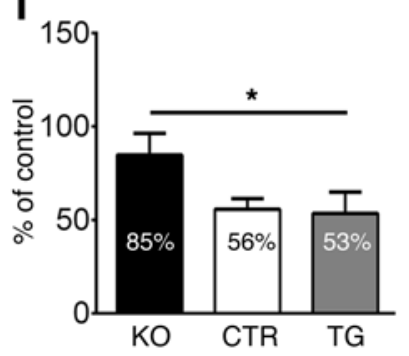

Figure 5. SORLA levels in WAT positively correlate with the extent of insulin-induced suppression of lipolysis. (A-F) Explants of gonadal WAT were generated from 4 genotype groups (SORLA KO, SORLA WT, SORLA WT/Cre, SORLA Tg) and treated in culture with the indicated substances. Thereafter, levels of FFAs in the tissues were determined. Panels B, D, F, and I display the concentration of FFAs for each genotype under untreated and treated conditions. Panels $\mathbf{A}, \mathbf{C}, \mathbf{E}$, and $\mathbf{H}$ give the extent of response for each genotype expressed as percentage change compared with the respective untreated genotype control (absolute values shown in panels B, D, F, and $\mathbf{I}$ and set to $100 \%$ in panels $\mathbf{A}, \mathbf{C}, \mathbf{E}$, and $\mathbf{H}$ ). As no difference in any of the parameters was observed comparing SORLA WT and SORLA WT/Cre animals, both genotypes were combined as control (CTR) lines. (A-D) Gonadal WAT from SORLA KO, SORLA Tg, and CTR (SORLA WT and SORLA WT/Cre) mice responded to treatment (48 hours) with NE and IPT (A and B) or with forskolin and IBMX (C and D) with a comparable increase in FFA production. (E and $\mathbf{F}$ ) Treatment with insulin for 60 minutes showed a genotype-dependent effect on FFA production with a strong reduction in SORLA Tg (51\% compared with control tissue), but a lesser decrease in SORLA KO (69\%) and CTR (65\%) explants. $n=6-14$ animal per genotype. ${ }^{*} P<0.05$; ${ }^{* *} P<0.01$; ${ }^{* * *} P<0.001$, unpaired Student's $t$ test $(\mathbf{A}, \mathbf{C}$, and $\mathbf{E})$; 1-way ANOVA (B, D, and $\left.\mathbf{F}\right)$. (C) Appearance of primary adipocytes derived from preadipocytes of the indicated Sorl1 genotypes (oil red $\mathbf{O}$ staining). Original magnification, $\times 40$. (H and I) Primary adipocytes of the indicated Sorl1 genotypes were treated with insulin for 30 minutes and the levels of released FFAs determined. $\mathbf{H}$ displays the concentration of FFAs for each genotype in the untreated and insulin-treated condition. I gives the extent of response for each genotype expressed as percentage of change compared with the respective untreated genotype control (set to 100\%). Insulin-dependent suppression of FFA production is stronger in cells expressing SORLA (CTR and Tg) than in adipocytes lacking the receptor (KO). 10-12 animals per genotype. ${ }^{*} P<0.05 ;{ }^{* *} P<0.01 ;{ }^{* *} P<0.001$, unpaired Student's $t$ test (H); 1 -way ANOVA (I).

SORLA Tg mice were treated with NE or the $\beta$-adrenergic receptor agonist isoproterenol (IPT), they displayed the same relative increase in FFA production (Figure 5, A and B). Also, simultaneous treatment with forskolin, which activates AC, and with 3-isobutyl1-methylxanthine (IBMX), which inhibits PDE3 $\beta$, stimulated FFA mobilization equally well in all 3 genotypes (Figure 5, C and D). While SORLA activity did not affect prolipolytic pathways, it had a profound effect on insulin-mediated inhibition of lipolysis. Thus, following acute treatment with insulin, levels of FFAs dropped in SORLA Tg tissues by $50 \%$, but only by about $30 \%$ in SORLA WT and SORLA KO (Figure 5, E and F). The ability of SORLA to promote insulin-mediated suppression of lipolysis was also seen in primary adipocytes differentiated from preadipocytes of gonadal adipose tissue pads of the various genotypes (Figure $5 G$ ). Again, acute stimulation with insulin suppressed release of FFAs more strongly in SORLA CTR and SORLA Tg compared with SORLA $\mathrm{KO}$ adipocytes (Figure 5, $\mathrm{H}$ and $\mathrm{I}$ ).

SORLA modulates insulin signaling in WAT and affects energy homeostasis in mice. Having established the ability of SORLA to promote insulin-mediated inhibition of lipolysis in explants, we tested the effect of this agonistic receptor action on insulin signaling in WAT in vivo. To do so, we acutely injected SORLA Tg, SORLA WT, SORLA WT/Cre, and SORLA KO mice with insulin and quantified the response to hormonal stimuli in WAT by Western blot analysis of IR pathway components. Within 15 minutes, all genotypes responded to insulin application with the expected increases in phosphorylated IR (p-IR) and AKT (p-AKT) levels (Supplemental Figure 9A). However, the extent of pathway acti- 

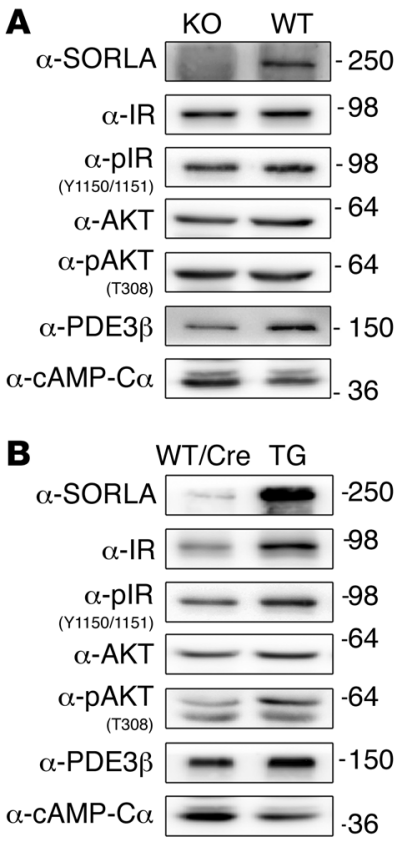
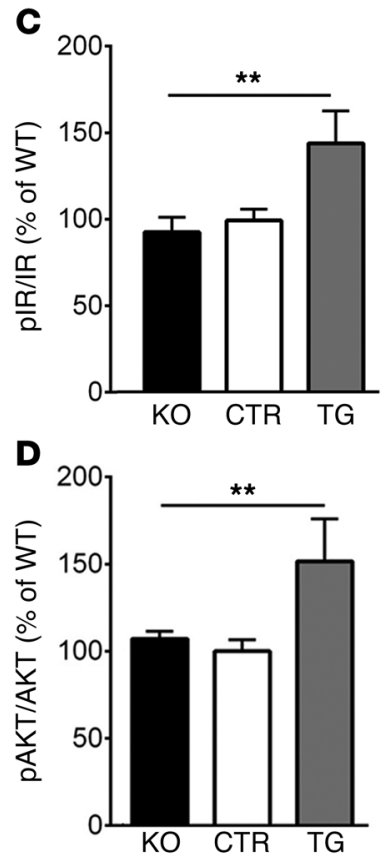

$\mathbf{E}$

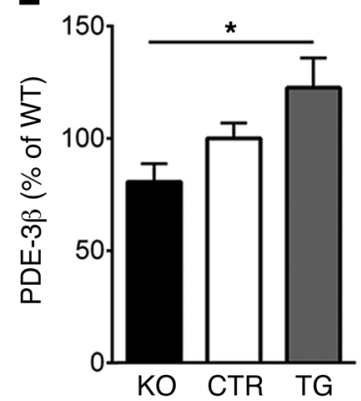

F

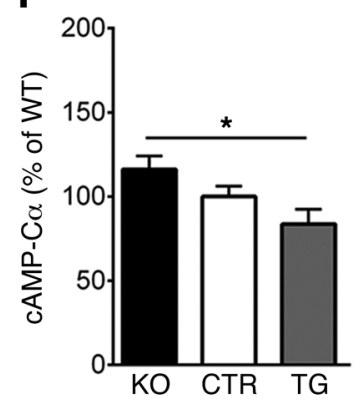

Figure 6. SORLA alters levels of IR signaling in WAT in vivo. SORLA KO, SORLA WT, SORLA WT/Cre, and SORLA Tg mice were injected with insulin (40 $\mu \mathrm{g} / \mathrm{kg}$ body weight), and gonadal WAT was dissected 15 minutes later. Levels of the indicated proteins in these tissues were determined by Western blotting (A and B) and by densitometric scanning of replicate blots (C-F). Protein levels in SORLA KO (A) or in SORLA Tg (B) mice were compared separately to their respective SORLA WT and SORLA WT/Cre control groups. Levels in both control lines were set to 100\% and jointly referred to as CTR in C-F. The amount of SORLA positively correlates with the relative levels of $p-I R$ ( $p$-IR/total IR; $\mathbf{C}$ ), p-AKT ( $p-A K T /$ total AKT; D), and PDE3 3 (E) in SORLA Tg as compared with SORLA CTR and SORLA KO mice. Also, SORLA levels negatively correlate with levels of cAMP-C $\alpha(\mathbf{F})$ in these mice. $n=15-32$ animals per genotype. ${ }^{*} P<0.05 ;{ }^{*} P<0.01$, 1-way ANOVA. The immunodetection of SORLA in $\mathbf{A}$ was derived from duplicate samples run on a separate gel.

vation following acute insulin stimulation was different and positively correlated with SORLA levels (Figure 6, A and B). Thus, the amounts of p-IR (Figure 6C) and p-AKT (Figure 6D) were higher in SORLA Tg than in SORLA CTR and SORLA KO mice. Also, PDE3 $\beta$ (Figure 6E) exhibited the highest levels in SORLA $\mathrm{Tg}$ and the lowest in SORLA KO. Conversely, levels of activated PKA were lowest in SORLA Tg and highest in SORLA KO (Figure $6 \mathrm{~F}$ ), in line with insulin signaling counteracting PKA-mediated activation of HSL. Levels of endogenous SORLA in SORLA WT mice significantly increased with acute insulin application (Supplemental Figure 9, A and B), a phenomenon caused by posttranscriptional mechanisms, as Sorl1 transcript levels remained unchanged (Supplemental Figure 9C). Changes in IR signaling rates were the cause of altered lipolytic activity in the various genotype groups, as other pathways affected by SORLA activity (e.g., bone morphogenetic protein signaling) (18) were not changed when comparing SORLA KO, SORLA CTR, and SORLA Tg mice (Supplemental Figure 10).

Altered levels of insulin signaling in adipose tissue affected the energy balance of the mice, as documented by analyzing metabolic parameters in SORLA KO and SORLA WT as well as in SORLA WT/Cre and SORLA Tg animals. As shown in Figure 7, basic metabolic rates, expressed as respiratory exchange ratio (RER), differed significantly, being highest in SORLA Tg, lowest in SORLA KO, and intermediate in SORLA WT and SORLA WT/ Cre (SORLA CTR). Relatively higher levels of the RER $\left(\mathrm{VCO}_{2} /\right.$ $\mathrm{O}_{2}$ ) indicate preferred carbohydrate oxidation, whereas lower levels indicate preferred lipid oxidation. Accordingly, our find- ings suggested a relative shift in metabolic fuel from carbohydrates in SORLA Tg to lipids in SORLA KO mice on normal chow. This conclusion was in line with the higher lipolytic activity in WAT of receptor-deficient as compared with receptor-overexpressing animals shown in Figure $4, \mathrm{~A}-\mathrm{C}$. Consistent with what has been reported for many mouse strains before $(19,20)$, the RER decreased in all mice on HFD with no obvious difference between SORLA genotype groups (Supplemental Figure 11A). Also, on HFD, the levels of activity and heat production were slightly decreased in SORLA CTR and SORLA Tg as compared with SORLA KO mice, likely reflecting the increased body weight in the obese strains (Supplemental Figure 11, C and D). Food intake was unchanged (Supplemental Figure 11B).

SORLA promotes insulin signaling to decrease lipolytic activity in cells. To further explore the cellular mechanism of SORLA in IR signaling, we used CHO cells, a model commonly used to study lipolysis (21). We compared parental $\mathrm{CHO}$ cells that do not express endogenous SORLA with CHO cells stably overexpressing the human receptor (CHO-S) (Supplemental Figure 12, $\mathrm{A}$ and $\mathrm{B})$. When grown in normal medium, CHO-S cells showed a significant accumulation of intracellular lipid droplets as compared with parental CHO cells (Figure 8A), a difference that was exacerbated by adding oleic acid to the culture medium (Figure 8B). Less lipid accumulation in CHO cells lacking SORLA resembled the phenotype seen in WAT of SORLA KO mice. As such, this observation supported the appropriateness of $\mathrm{CHO}$ cells for studying SORLA action in lipolysis. Our conclusion was confirmed by documenting decreased lipolytic activity in CHO-S as 

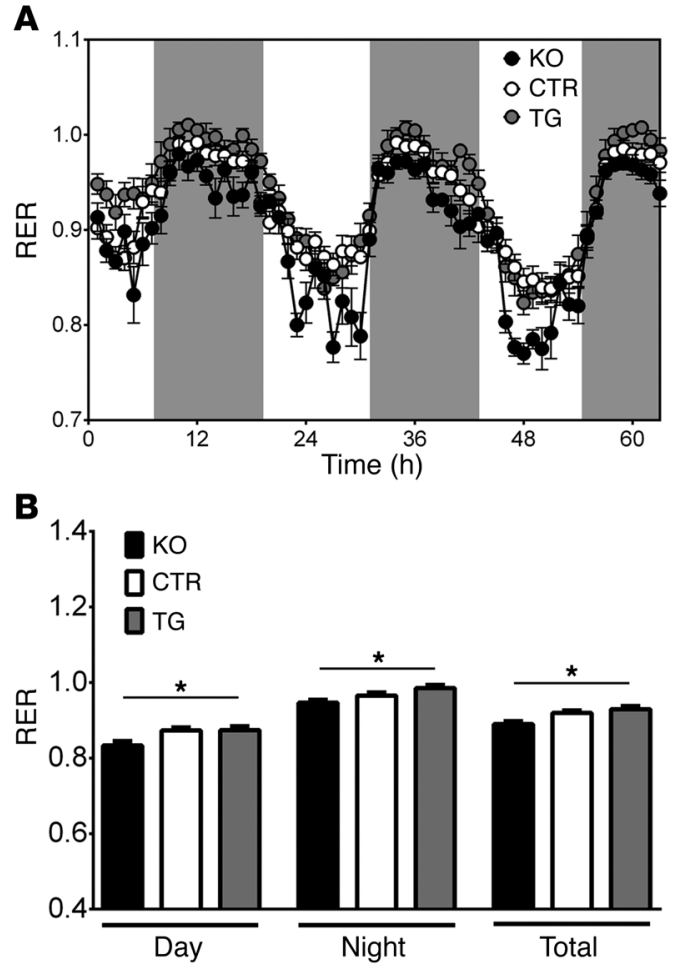

compared with parental CHO cells (Figure 8C). Decreased lipolysis coincided with increased levels of PDE3 $\beta$ and decreased levels of activated PKA in CHO-S (Figure 8, D and E). These differences in insulin signaling components were due to an increased sensitivity of CHO-S cells to insulin stimulation. This effect was shown by treatment of cells with insulin for 15 minutes, reducing lipolysis more strongly in $\mathrm{CHO}-\mathrm{S}$ than in $\mathrm{CHO}$ cells (Figure $8 \mathrm{~F})$. While insulin treatment caused the expected decreases in activated PKA levels in CHO-S cells, the response in $\mathrm{CHO}$ cells lacking SORLA was blunted, as activated PKA levels remained unchanged compared with those in $\mathrm{CHO}$ cells not treated with insulin (Figure 8, $\mathrm{G}$ and $\mathrm{H}$ ).

SORLA interacts with the IR to promote its cell surface exposure. Next, we investigated the molecular interaction of the endogenous IR with SORLA in CHO-S cells. As shown in Supplemental Figure 12C, SORLA colocalized with the endogenous IR in these cells. Moreover, both proteins directly interacted, as documented by coimmunoprecipitation of the IR with anti-SORLA antiserum from CHO-S cells (Figure 9A). Interaction with SORLA strongly increased the surface exposure of the IR. This effect was documented by biotinylation of surface proteins in $\mathrm{CHO}$ and $\mathrm{CHO}-\mathrm{S}$ cells and by semiquantitative Western blot analysis of the amount of biotinylated IR precipitated from both cell lines. While the amount of total IR in the CHO-S lysate was even slightly decreased, the amount of receptor on the cell surface was increased by $60 \%$ as compared with that in $\mathrm{CHO}$ (Figure 9, B and C). Enhanced surface exposure of the IR in the presence of SORLA was substantiated by comparing immunosignals for the IR in CHO-S and CHO cells not permeabilized with Triton X-100 (Supplemental Figure 12D; panel without Triton X-100).

The functional interaction of SORLA with the IR seen in CHO-S cells was confirmed for the endogenous proteins in adipo-
Figure 7. SORLA activity affects basic metabolic rates in vivo. (A) Basic metabolic rates were determined in SORLA KO, SORLA WT, SORLA WT/ Cre, and SORLA Tg mice and expressed as RER. As no statistically significant difference were noted comparing SORLA WT and SORLA WT/Cre animals, both genotypes were combined as control (CTR) group. (B) The RER is displayed separately for day ( 6 am- $6 \mathrm{pm})$ and night ( $6 \mathrm{pm}-6 \mathrm{am})$ as well as combined. The data show a significant increase in RER with increasing levels of SORLA when comparing SORLA KO, SORLA CTR, and SORLA Tg animals. $n=5-7$ animals per genotype. ${ }^{*} P<0.05$, 1-way ANOVA.

cytes. Thus, the physical interaction of endogenous SORLA with the IR was documented by coimmunoprecipitation of the IR with anti-SORLA antiserum from WAT of SORLA WT but not from SORLA KO animals (Figure 9D). To assess the effect of endogenous SORLA on the surface exposure of the IR, we differentiated murine embryonic fibroblasts (MEFs) from SORLA WT and SORLA KO mice to adipocytes. While SORLA was not expressed in fibroblasts, differentiation into adipocytes resulted in massive induction of receptor expression in SORLA WT cells (Supplemental Figure 13). Coexpression with SORLA resulted in significantly higher levels of the IR being exposed on the surface of SORLA WT adipocytes compared with that of SORLA KO cells, as shown by surface biotinylation experiments (Figure 9, E and F). Finally, enhanced cell surface exposure of the IR in adipocytes overexpressing SORLA was documented by immunostaining of SORLA WT/Cre and SORLA Tg primary adipocytes not permeabilized with detergent (Supplemental Figure 12E).

SORLA-mediated sorting prevents lysosomal catabolism and facilitates cell surface reexposure of the IR. Increased surface exposure of the endogenous IR in CHO-S cells and primary adipocytes was caused by altered trafficking of the IR in the presence of the sorting receptor SORLA. As seen in cells permeabilized with Triton X-100 prior to immunostaining, the IR showed a predominant perinuclear (Golgi like) pattern in CHO-S, but a dispersed vesicular pattern in CHO cells lacking SORLA (Supplemental Figure 12D). Altered sorting of the IR by SORLA was confirmed by costaining of the IR with markers of endocytic and secretory compartments (Supplemental Figure 14A) and by determining the extent of colocalization by Pearson's correlation coefficient (Supplemental Figure 14B). Compared with $\mathrm{CHO}$ cells, the presence of SORLA in CHO-S increased the levels of the IR in the trans-Golgi network (TGN) (marker VTI1B) and in Golgi-destined vesicles (marker AP1). It also raised IR levels in vesicles directing cargo to the cell surface, including secretory vesicles (marker VTI1A) and recycling endosomes (marker RAB11). In contrast, while the levels of the IR in CHO-S were increased in early endosomes (marker EEA1), no increase was seen in RAB9-positive late endosomes (Supplemental Figure 14B). These data indicate that SORLA promotes recycling of internalized IR molecules via the Golgi to the cell surface, avoiding a late endosomal/lysosomal fate for the IR.

Binding of insulin to the IR induces internalization and lysosomal degradation of the receptor in adipocytes, a means for downregulating this signaling pathway after stimulation $(22,23)$. To explore the consequence of insulin-dependent sorting of the IR in the presence or absence of SORLA, we tagged IR molecules on the surface of $\mathrm{CHO}$ and $\mathrm{CHO}-\mathrm{S}$ cells at $4^{\circ} \mathrm{C}$ using anti-IR antiserum. Subsequently, insulin was added to the medium and the cells were shifted to $37^{\circ} \mathrm{C}$ to track IR internalization by immuno- 
A

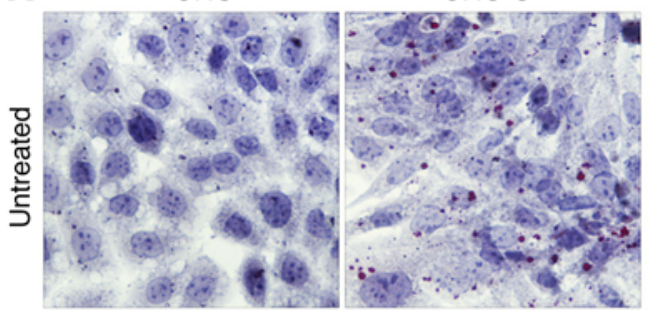

C

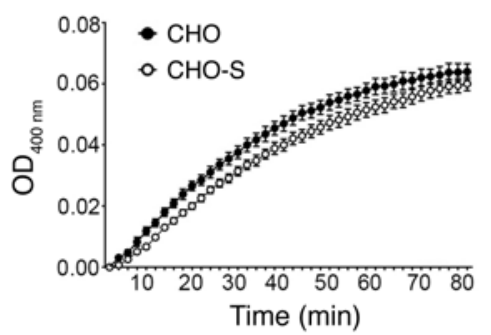

$\mathbf{F}$

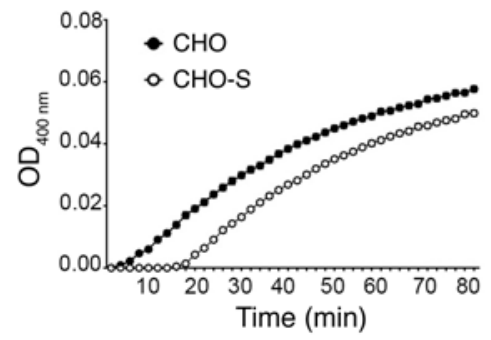

D

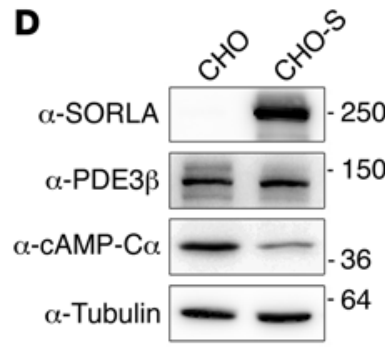

G

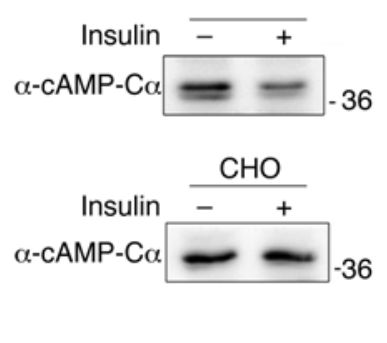

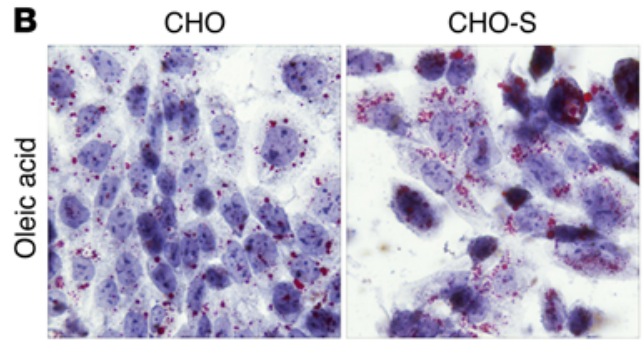
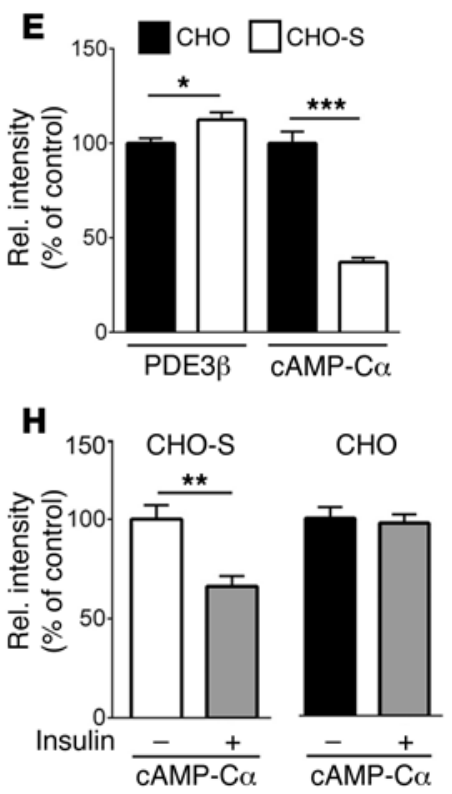

Figure 8. SORLA enhances IR signaling and lipid accumulation in CHO cells. (A and B) Oil red O staining of parental CHO cells and CHO-S cells. Cells grown in normal medium (A) or in medium containing $100 \mu \mathrm{M}$ oleic acid (B). Accumulation of intracellular lipid droplets (red) is more pronounced in CHO-S as compared with $\mathrm{CHO}$ cells under both conditions. Original magnification, $\times 40$. (C) Lipase activity in $\mathrm{CHO}$ and $\mathrm{CHO}-\mathrm{S}$ cells expressed as percentage of signal increase at $\mathrm{OD}_{400 \mathrm{~nm}}$ over time. Lipolytic activity is significantly lower in CHO-S as compared with CHO cells. $n=10$. $P<0.05$ for genotypes; $P<0.05$ for interaction, matched 2-way ANOVA. ( $\mathbf{D}$ and E) Densitometric scanning of replicate Western blots (as exemplified in $\mathbf{D}$ ) documents significantly increased levels of PDE3 $\beta$ and decreased levels of cAMP- $\alpha \alpha$ in CHO-S as compared with CHO cells. $n=6-11 .{ }^{*} P<0.05$; ${ }^{* *} P<0.001$, unpaired Student's $t$ test. (F) Lipase activity in $\mathrm{CHO}$ and $\mathrm{CHO}-\mathrm{S}$ cells treated with $10 \mu \mathrm{g} / \mathrm{ml}$ insulin for 15 minutes (expressed as percentage increase at $\mathrm{OD}_{400 \mathrm{~nm}}$ ). The enzyme activity is significantly lower in CHO-S as compared with CHO cells. $n=10$. $P<0.001$ for genotypes; $P<0.001$ for interaction, matched 2-way ANOVA. (G and $\mathbf{H}$ ) CHO and CHO-S cells were treated with control medium or with medium containing $10 \mu \mathrm{g} / \mathrm{ml}$ insulin for 15 minutes and the amount of cAMP-C $\alpha$ in cell extracts determined by Western blotting thereafter. Representative Western blots are shown in $\mathbf{G}$. The results of densitometric scanning of replicate blots are given in $\mathbf{H}$. CHO-S cells respond to insulin treatment with decreased levels of CAMP- $C \alpha$, whereas CHO cells lacking SORLA do not respond to insulin stimulation. $n=6 .{ }^{* *} P<0.01$, unpaired Student's $t$ test.

cytochemistry. Within 20 minutes, application of insulin induced a shift of surface-localized IR molecules to intracellular compartments in $\mathrm{CHO}$ and CHO-S cells (Figure 10A). While the kinetics of internalization was comparable in both cell types, the fate of the internalized IR molecules was quite different, as documented by costaining for subcellular marker proteins. As exemplified for RAB11 by immunofluorescence microscopy in Figure 10A and quantified for RAB11 and RAB9 in Figure 10, B and C, the presence of SORLA promoted accumulation of the IR in recycling endosomes (RAB11), but reduced IR targeting to late endosomes (RAB9). The very same activity of SORLA was also observed in primary adipocytes tagged for endogenous IR molecules on the cell surface using anti-IR antiserum at $4^{\circ} \mathrm{C}$ (Figure 11A). Within 30 minutes after insulin treatment and shift to $37^{\circ} \mathrm{C}$, surface-tagged IR molecules preferentially routed to RAB11-positive recycling endosomes in SORLA Tg adipocytes, but to RAB9-positive late endosomes in SORLA KO cells (Figure 11, B and C).
Prevention of IR targeting to late endosomes/lysosomes by SORLA markedly increased the half-life of this receptor following stimulation by insulin. This effect was documented by biotinylation of the IR on the surface of $\mathrm{CHO}$ and CHO-S cells and by quantification of the tagged receptor molecules at various time points after stimulation of the cells with insulin by Western blotting. As shown in Figure 12, the amount of IR molecules originating from the cell surface decreased significantly faster in $\mathrm{CHO}$ as compared with CHO-S cells. Decay of IR molecules was blocked to a similar extent in both cell lines by application of a lysosomal inhibitor cocktail (leupeptin, pepstatin, and chloroquine).

Taken together, our data from $\mathrm{CHO}$ cells and primary adipocytes uncovered the molecular mechanism whereby SORLA acts as a unique sorting factor that redirects internalized IR molecules back to the cell surface, thereby preventing lysosomal catabolism of the IR and strengthening insulin signal reception in adipose tissue. 
A

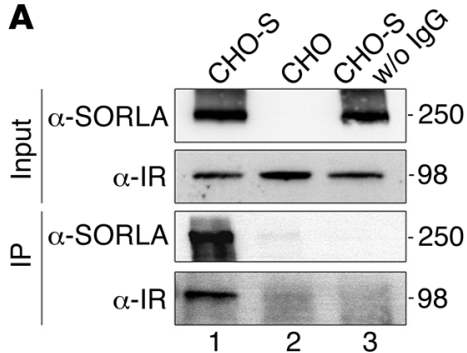

D

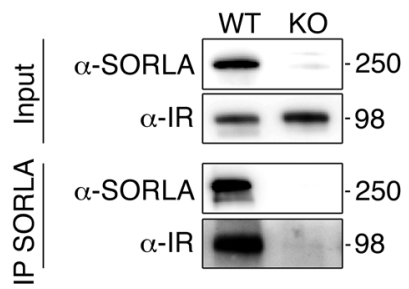

B

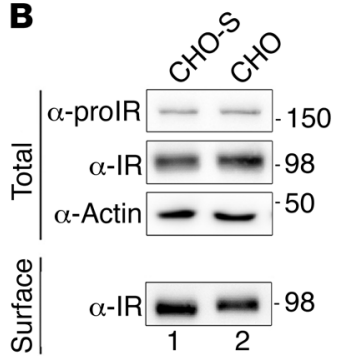

E

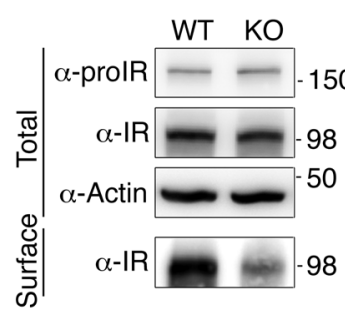

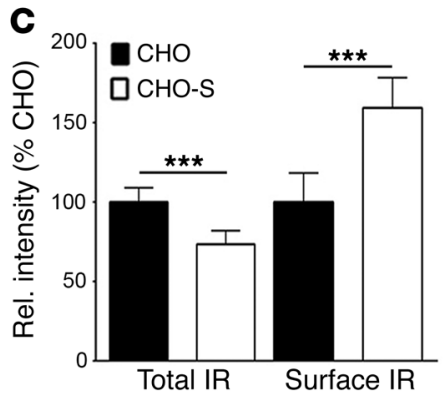

F $\quad \mathrm{KO}$

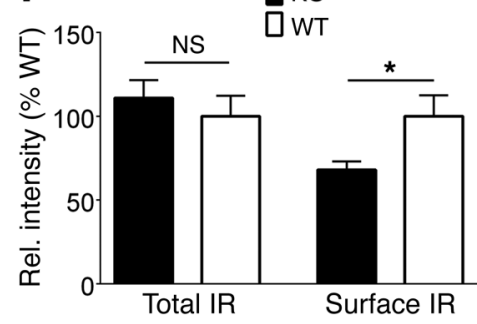

Figure 9. SORLA interacts with the IR to increase cell surface exposure. (A) Parental CHO cells and CHO-S cells were subjected to immunoprecipitation using anti-SORLA antiserum. Input represents Western blot analyses of cell lysates prior to immunoprecipitation. IP depicts SORLA and IR in the precipitate. Precipitation of SORLA from CHO-S (lane 1) results in coimmunoprecipitation of the IR. No (co)immunoprecipitation of SORLA and IR is seen in parental CHO cells lacking SORLA (lane 2) or in CHO-S cells without addition of anti-SORLA IgG (w/o IgC; lane 3). (B and C) Replicate layers of CHO and CHO-S cells were treated with sulfo-NHS-S-S-biotin and biotinylated surface proteins precipitated by streptavidin beads. The amount of IR in total cell lysates (total levels) and in the biotinylated fraction (surface) was determined by Western blotting (B). Cell surface levels of the IR are increased in CHO-S as compared with parental CHO cells (relative levels set to $100 \%$ ), as shown by densitometric scanning of replicate experiments (C). $n=10$. ${ }^{* * *} P<0.001$, unpaired Student's $t$ test. (D) Coimmunoprecipitation of endogenous IR with endogenous SORLA from gonadal WAT of SORLA WT but not SORLA KO mice using anti-SORLA IgG (IP SORLA). Input documents levels of SORLA and IR in SORLA WT and SORLA KO WAT prior to immunoprecipitation. (E and F) MEFs from SORLA WT and SORLA KO mice were differentiated to adipocytes. Replicate layers of adipocytes were treated with sulfo-NHS-S-S-biotin and biotinylated surface proteins precipitated by streptavidin beads. The amount of IR in total cell lysates (total) and in the biotinylated fraction (surface) was determined by Western blotting (E) and by densitometric scanning of replicate experiments (F). The total levels of the IR are unchanged, but the cell surface levels decreased in SORLA KO as compared with SORLA WT adipocytes (relative levels set to $100 \%$ ). $n=6-7$ per genotype. ${ }^{*} P<0.05$, unpaired Student's $t$ test.

\section{Discussion}

We have identified a regulatory pathway whereby SORLA facilitates cell surface exposure of the IR to increase insulin signaling and to reduce lipolytic activity in adipocytes. In mice, decreased lipolysis in WAT in the presence of SORLA promotes the accumulation of triacylglycerides and exacerbates diet-induced obesity, while loss of SORLA activity causes excessive lipolysis and a reduction in WAT mass. Genome-wide association of SORL1 with obesity (2) and the correlation of SORLA protein levels in adipose tissue with BMI in human subjects shown here underline the clinical relevance of this receptor for disorders of the human metabolism.

SORLA is a sorting receptor for the IR. SORLA is a sorting receptor that shuttles between endosomes and the TGN to sort target proteins between endocytic and secretory compartments of the cell. Shuttling of SORLA is guided by the adaptors GGA, PACS1, and retromer, which bind to the cytoplasmic tail of the receptor and direct its movement from endosomes to the TGN and back $(24,25)$. The relevance of SORLA-mediated protein sorting has best been elucidated for APP in AD. SORLA-mediated retrieval of APP from early endosomes to the TGN reduces the production of neurotoxic $A \beta$ peptides, the cause of neurodegenerative processes in $\mathrm{AD}(6,7)$. This protective action renders SORLA one of the major risk factors for sporadic AD known to date (8). The identification of mutations in SORL1 in cases with autosomal-dominant forms of the disease suggests SORLA as a familial AD gene as well $(11,26)$. Now, our studies identified the IR as a target for SORLA-mediated protein sorting in adipocytes. Similarly to APP, interaction of the IR with SORLA redirected internalized IR molecules from early endosomes to the TGN and to cell surface-destined recycling compartments in the presence of insulin stimuli (Figures 10 and 11). This retrieval pathway reduced the amount of IR molecules delivered to lysosomal catabolism (Figure 12) and increased the fraction of receptors reexposed on the cell surface (Figure 9).

SORLA-mediated sorting of the IR promotes insulin action in cells. Cellular trafficking of the IR has long been recognized as a mechanism to control insulin signaling (reviewed in ref. 27). Following binding of the hormone on the cell surface, complexes of IR and insulin undergo endocytosis that serves 2 purposes. First, it delivers receptor-ligand complexes to endosomes, a prerequisite for signal transduction. Second, it moves receptor-ligand complexes to lysosomal compartments for catabolism, a means of downregulating signal reception. In CHO cells, SORLA activity promotes insulin-dependent suppression of lipolysis and accelerates lipid accumulation (Figure 8). The same mechanism is operable in adipocytes. In vivo, the presence of SORLA promotes insulin-dependent signaling in WAT (Figure 6) and stimulates insulin-induced suppression of lipolysis in adipose tissue explants (Figure 5, E and F) and primary adipocytes (Figure 5, $\mathrm{H}$ and I). Other pathways in control of lipolysis (e.g., through catecholamines) are not influenced by SORLA (Figure 5, A-D), 


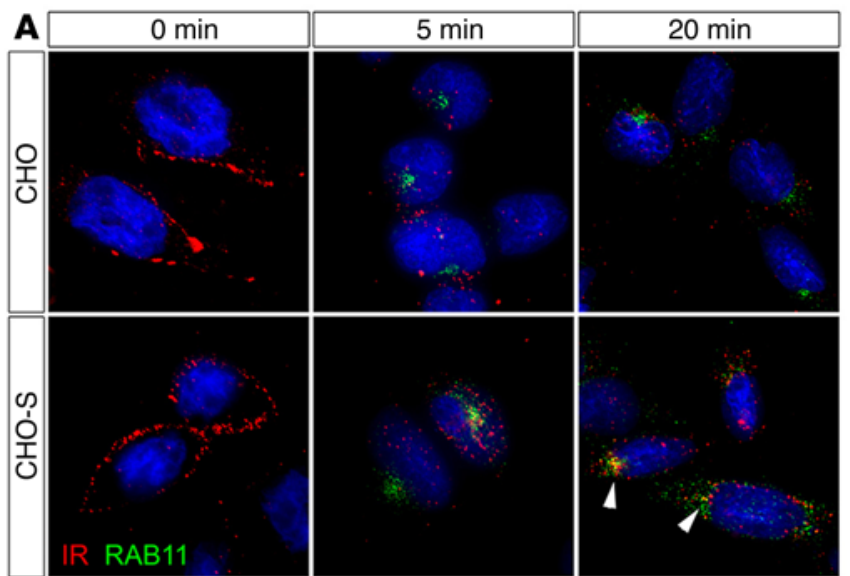

B

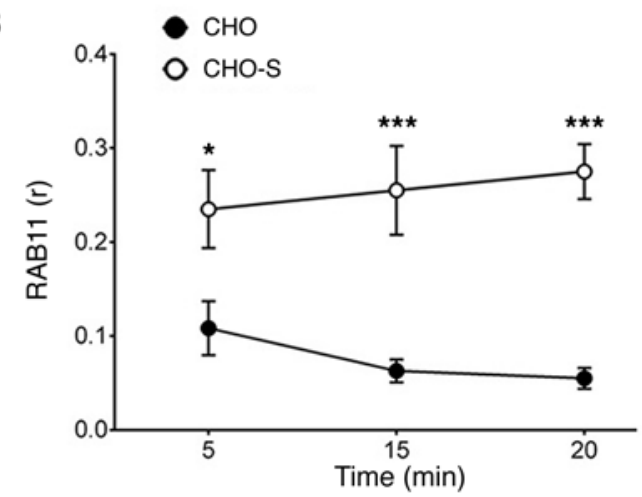

C

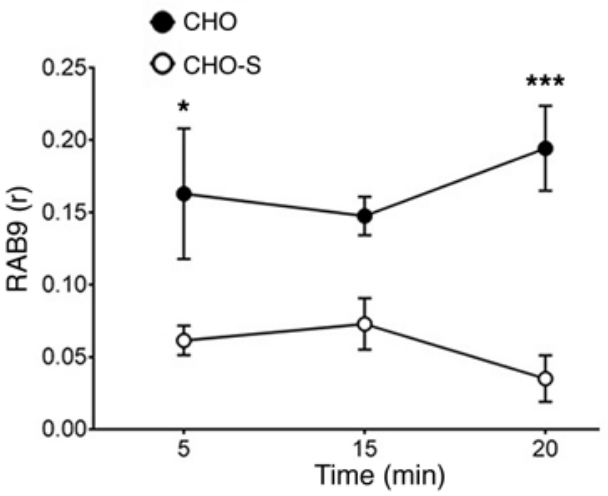

documenting the specificity of SORLA for insulin action in this cell type. Our data are most consistent with a model whereby SORLA directs the IR from early endosomes to recycling compartments (marked by VTI1A and RAB11) to prevent lysosomal breakdown of receptor molecules and to enhance their reexposure at the cell surface. Because SORLA increases the amount of IR in early endosomes (marked by EEA1), it may also facilitate signal transduction by promoting internalization of IR/ligand complexes into signaling endosomes.

SORLA determines WAT mass by affecting lipolysis rates in adipocytes. SORLA is expressed in a number of tissues relevant to energy metabolism, including the liver, skeletal muscle, and adipose tissue (refs. 28, 29, and Supplemental Figure 3A). Because SORLA binds apolipoproteins $(30,31)$ and LpL (10) in vitro, a role for this receptor in systemic lipoprotein metabolism had been proposed. Now, our studies in mouse models with loss or overexpression of this receptor substantiate the relevance of
Figure 10. SORLA promotes cell surface recycling of internalized IRs in CHO cells. (A) Replicate layers of $\mathrm{CHO}$ and $\mathrm{CHO}-\mathrm{S}$ cells were blocked at $4^{\circ} \mathrm{C}$ and cell surface-exposed IR molecules tagged with anti-IR IgC (0 minutes). Subsequently, $10 \mu \mathrm{g} / \mathrm{ml}$ insulin was added to the medium and cells shifted to $37^{\circ} \mathrm{C}$. At time points 5, 15, and 20 minutes, the cells were fixed and costained for the tagged IR molecules (red) and recycling endosomal marker RAB11 (green). Colocalization of both proteins is seen in CHO-S (arrowheads) but not $\mathrm{CHO}$ cells after 20 minutes of chase. Original magnification, $\times 60$. (B and $\mathbf{C}$ ) Experiments as exemplified in $\mathbf{A}$ were performed and the colocalization of the IR with RAB11 (B) and RAB9 (C) evaluated by Pearson's correlation coefficient $(r)$. The IR preferentially colocalized with RAB11 in CHO-S as compared with $\mathrm{CHO}$ cells. In contrast, colocalization of the IR with late endosomal marker RAB9 was reduced in $\mathrm{CHO}-\mathrm{S}$ as compared with $\mathrm{CHO}$ cells. $n=20-30$ cells. ${ }^{*} P<0.05$; ${ }^{* *} P<0.001$, 2-way ANOVA.

SORLA for lipid homeostasis in vivo. However, contrary to previous hypotheses, our studies failed to support the significance of SORLA for systemic lipoprotein metabolism, as lipoprotein profiles were indistinguishable when comparing SORLA WT, SORLA WT/Cre, SORLA KO, and SORLA Tg mice on normal chow. Also, LpL activity in plasma and in tissues was unchanged when comparing Sorl1 genotypes. Rather than affecting the level of circulating lipoproteins, our studies identified the involvement of SORLA in control of adipose tissue mass. A positive correlation of SORLA levels with white adipose mass was seen in mice on normal chow (Figure $1 \mathrm{C}$ and Figure $2 \mathrm{~B}$ ) and was greatly exacerbated by feeding animals with HFD (Figure 1A and Figure 2C). Also, transcript and protein levels for SORL1 in WAT correlated with BMI in humans (Figure 3).

Conceivably, the loss of expression of SORLA in many tissues that normally express the receptor may contribute to the lean phenotype seen in SORLA KO animals. However, tissuespecific overexpression of the receptor in adipocytes of SORLA $\mathrm{Tg}$ mice resulted in features that were the opposite of those seen in SORLA KO animals, including enhanced fat mass (Figure 2) and reduced lipolytic activity (Figure 4) as well as increased insulin-dependent signaling and reduced rates of lipolysis in primary adipocytes and adipose tissue explants (Figure 5) as well as in vivo (Figure 6). These findings strongly argue that loss of SORLA activity in adipocytes is the cause for the reduced adipose tissue mass in SORLA KO animals.

All available evidence from studies in primary adipocytes, in murine and human WAT, and in in vivo experimentation in this study is consistent with a model whereby SORLA modulates the activity of the IR in adipocytes and determines the extent of insulin-induced suppression of lipolysis. The progressive decrease in lipolytic activity from SORLA KO to SORLA CTR to SORLA Tg mice (Figure $4, \mathrm{~A}^{-} \mathrm{C}$ ) coincides with a concomitant shift in the usage of carbohydrates over lipids as an energy source, as shown by the change in RER (Figure 7), documenting the impact of SORLA levels on energy homeostasis in vivo.

Recently, a role for the soluble ectodomain of SORLA (called sLR11) in control of thermogenesis in mice has been reported. Specifically, sLR11 is proposed to act via the BMP receptor pathway to suppress browning of WAT (18). Possibly, SORLA may assume different roles in control of systemic metabolism, including action as a humoral factor. However, our data unambiguously document a cell-autonomous function for the full-length recep- 

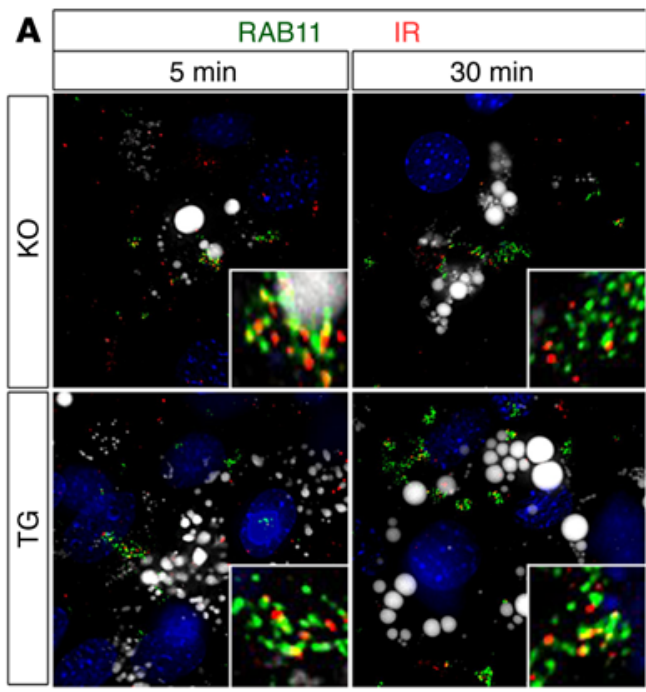

B

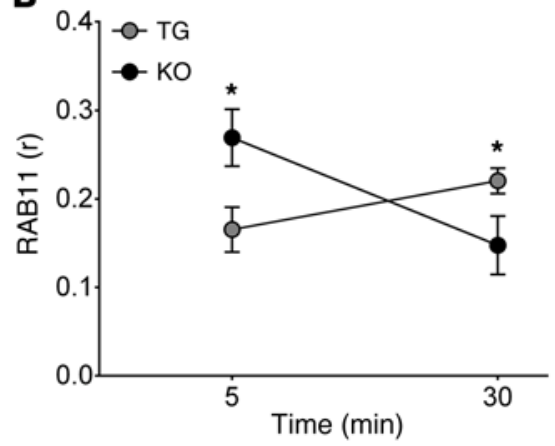

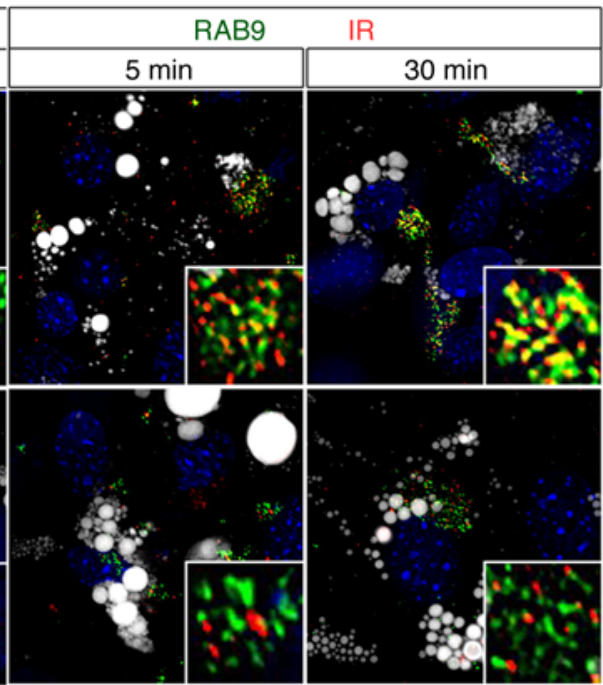

C

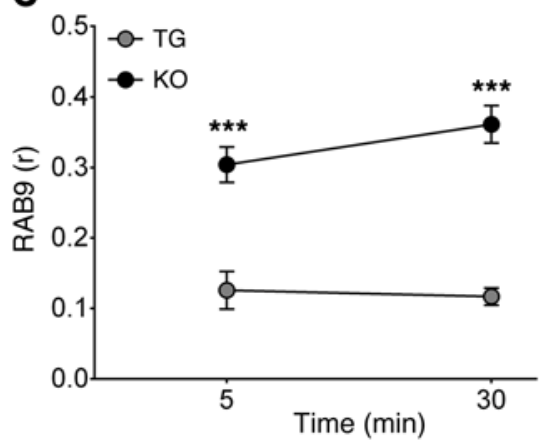

Figure 11. SORLA promotes recycling of internalized IR to the cell surface in primary adipocytes. (A) Replicate layers of primary adipocytes from SORLA KO and SORLA Tg mice were blocked at $4^{\circ} \mathrm{C}$ and cell surface-exposed IR molecules tagged with anti-IR IgC. Subsequently, $10 \mu \mathrm{g} / \mathrm{ml}$ insulin was added to the medium and cells shifted to $37^{\circ} \mathrm{C}$. At time points 5 and 30 minutes, the cells were costained for the tagged IR molecules (red) and markers RAB11 or RAB9 (green). In SORLA KO adipocytes, the IR colocalizes preferentially with RAB9 (see merged images in the insets). In contrast, in SORLA Tg adipocytes, the IR colocalizes with RAB11 after 30 minutes of chase. Both overview and inset images were acquired at $\times 60$ magnification. Overview images were shrunk to $30 \%$ of original size. ( $\boldsymbol{B}$ and $\mathbf{C}$ ) Experiments as exemplified in $\mathbf{A}$ were performed and the colocalization of the IR with RAB11 (B) and RAB9 (C) evaluated by Pearson's correlation coefficient $(r)$. The IR preferentially colocalized with RAB11 in SORLA Tg, but with RAB9 in SORLA KO adipocytes. $n=20-30$ cells per genotype. ${ }^{*} P<0.05 ;{ }^{* *} P<0.001,2$-way ANOVA. tor in control of body weight and the incidence of obesity. This conclusion is supported by unchanged rates of transcription of genes proposed to act downstream of sLR11 in control of thermogenesis (18) in experimental conditions described here (Supplemental Figure 10).

$S O R L A$, a regulator of energy balance and risk factor for human obesity. GWAS uncovered SORL1 as a gene associated with metabolic phenotypes in humans and mouse models. In the longitudinal Bogalusa Heart Study (2), SORL1 emerged as a top hit associated with the risk of obesity in children as they advance through adulthood. In the analysis of obesity traits in inbred strains of mouse, Sorl1 predicted body fat percentage (9), while global transcriptome analysis in mice with diet-induced obesity identified Sorl1 as an adipocyte-specific gene responsive to caloric restriction (32). Massive induction of Sorl1 expression in MEFs, as they differentiate to adipocytes, supports a pivotal role for this receptor in adipocyte function. Expression of SORL1 is correlated with BMI in humans (Figure 3) and promoted by insulin in adipocytes (Supplemental Figure 9), reinforcing the relevance of this receptor for metabolic pathways in fat tissue.

What is the evidence that the ability of SORLA to modulate insulin-dependent rates of lipolysis in WAT explains the association of SORL1 with obesity in humans and mice? Foremost, this assumption is supported by our findings that the level of this receptor in SORLA KO, SORLA CTR, and SORLA Tg mice inversely correlates with the ability to mobilize lipids from adipocytes (Figure 4) and predict fat tissue mass (Figure 2). This effect of SORLA is already evident on normal chow and underlies the exacerbation of diet-induced obesity seen on a HFD. Disturbances in glucose handling (hyperinsulinemia, hyperglycemia) as well as advanced tissue pathology (adipose tissue hypertrophy, liver steatosis) in SORLA Tg mice on a HFD are secondary consequences of overt obesity in these animals (Supplemental Figures 6 and 7). They do not reflect a primary effect of SORLA on glucose homeostasis, as hypersensitivity to insulin seen in adipocytes of the SORLA Tg line is expected to facilitate rather than inhibit insulin action on glucose uptake.

The importance of insulin in determining lipolysis rates and, thereby, fat mass is underscored by mouse models and humans with alterations in the insulin pathway in adipocytes. For example, targeted disruption of the gene encoding PDE3 $\beta$, the PDE acting downstream of the IR in adipocytes, blocks insulin inhibition of lipolysis, thereby causing excessive lipolytic activity and a decrease in WAT mass in mice. The effect of PDE3 $\beta$ deficiency is specific for insulin-dependent control of lipolysis, as it does not alter glucose handling by adipocytes (33). Phosphatase and tensin homolog (PTEN) is an effector molecule that antagonizes insulin action through inhibition of AKT. Haploinsufficiency for PTEN in humans results in insulin hypersensitivity in adipose tissue and in obesity (34), while overexpression of the protein in mice reduces body fat accumulation (35). Apart from these observations in monogenic traits, epidemiological studies also show reduced lipolytic activity in adipose tissue of obese individuals (36-38), suggesting impaired mobilization of fat stores as 
A
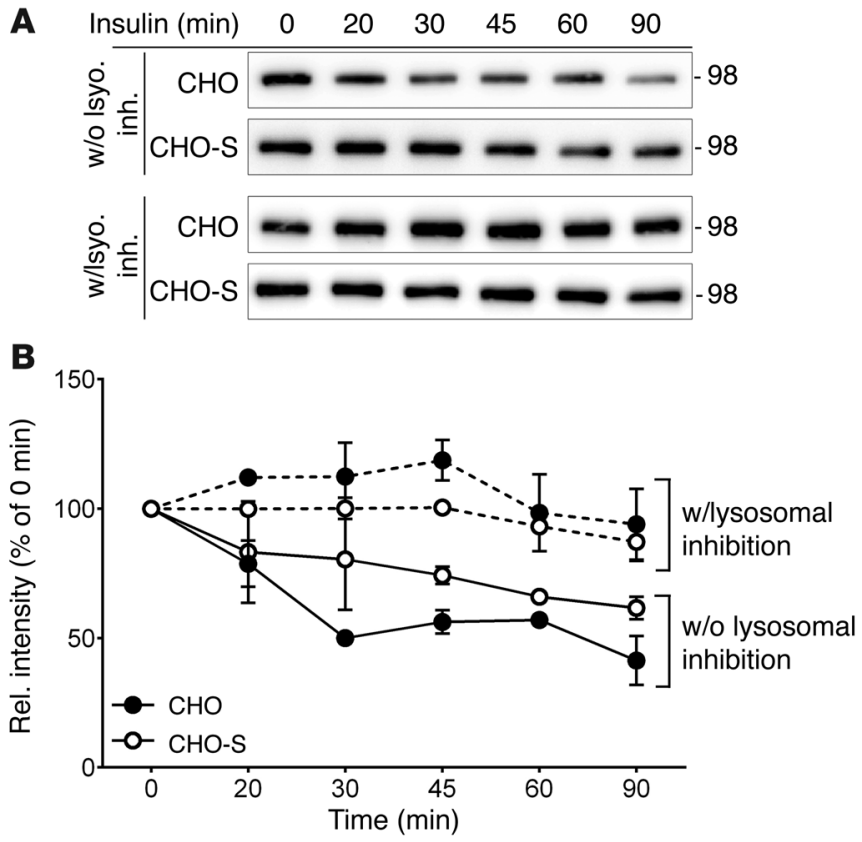

a primary risk factor for the development of excess body fat. Our studies have substantiated the relevance of lipolytic rate for risk of obesity and a central role for SORLA as a sorting factor for the IR in this pathology. Overall, our findings also document a genetic link between obesity and neurodegeneration to converge on this sorting receptor pathway.

\section{Methods}

Study participants. For quantitative PCR (q-PCR) analysis, paired samples of visceral whole adipose tissue were obtained from 362 individuals (246 women and 116 men). The age ranged from 19 to 93 years. BMI was calculated by weight $(\mathrm{kg})$ divided by square of height $(\mathrm{m})$. All adipose tissue samples were collected during open or laparoscopic abdominal surgery, immediately frozen in liquid nitrogen, and stored at $-80^{\circ} \mathrm{C}$. Where sufficient tissue was available for protein extraction, specimens were also used for SORLA protein quantification by ELISA.

Animal experimentation. Mice with targeted disruption of Sorl (Sorl1 $\left.{ }^{--}\right)$(6) or carrying a cDNA encoding WT human SORLA in the Rosa26 locus (Sorl1 ${ }^{+/+}$Rosa26 $6^{\text {TgSORLIWT }}$ ) (11) have been described. Sorl1 ${ }^{+/+}$Rosa $26^{\text {TgSORLIWT }}$ animals were crossed with the $\mathrm{Tg}^{\text {(Adipoq-Cre)1Evdr/I }}$ line (JAX 010803; The Jackson Laboratory) to direct SORLA overexpression to adipocytes. All lines were kept by breeding of heterozygous animals, and all studies were performed comparing littermates. Specifically, Sorl1 ${ }^{-1-}$ (referred to as SORLA KO) mice were compared with Sorl1 ${ }^{+/+}$littermates (referred to as SORLA WT). Sorl1 ${ }^{+/+}$Rosa26 $6^{\text {TgSORLIWT }} \mathrm{Tg}^{\text {(Adipoq-Cre)1Evdr/J }}$ (referred to as SORLA Tg)

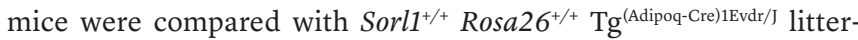
mates (referred to as SORLA WT/Cre). No discernible phenotypic differences were seen when comparing SORLA WT and SORLA WT/Cre control groups. Thus, where applicable, both strains were combined into 1 control cohort (referred to as SORLA CTR) when testing Sorl1 gene-dosage effects in SORLA KO versus SORLA Tg genotypes. Animals were kept on a standard mouse chow $(4.5 \%$ crude fat, $39 \%$ carbohydrates) or a HFD (35\% crude fat, $10 \%$ car-
Figure 12. SORLA reduces lysosomal degradation of internalized IR molecules. (A) Cell surface proteins in cultures of $\mathrm{CHO}$ and $\mathrm{CHO}-\mathrm{S}$ cells were biotinylated. Subsequently, $10 \mu \mathrm{g} / \mathrm{ml}$ insulin was added to the cells and the biotinylated proteins precipitated from cell lysates at the indicated time points using streptavidin. The amount of endogenous IR in the precipitate was determined by Western blot analysis. Where indicated, the lysosomal inhibitor (lyso. inh.) cocktail of leupeptin, pepstatin, and chloroquine was absent (w/o) or included ( $w /)$ in the cell medium. (B) Densitometric scanning of replicate blots as exemplified in $\mathbf{A}$ documents faster turnover of IR molecules originating from the cell surface in $\mathrm{CHO}$ as compared with $\mathrm{CHO}-\mathrm{S}$ cells. No difference in half-life of the IR was observed when the lysosomal activity in the $\mathrm{CHO}$ and $\mathrm{CHO}-\mathrm{S}$ cells was blocked by the lysosomal inhibitors. $P<0.05$ for genotypes (w/o lysosomal inhibition); not significant for genotypes (with lysosomal inhibition). Unmatched 2-way ANOVA.

bohydrate). All experiments were performed in male mice from 30 to 50 weeks of age. Details about the metabolic studies in mice are given in the Supplemental Methods.

Lipid analyses. Lipid levels in plasma and tissue were determined by Triacylglyceride Colorimetric Assay Kit (Cayman Chemical Company), Amplex Red Cholesterol Assay Kit (Molecular Biotechnology), or Free Fatty Acid Quantification Kit (BioVision) according to the manufacturers' protocols. Lipoprotein profiles were characterized using FPLC (TNO Metabolic Health Research). Released glycerol was determined using the Free Glycerol Kit (Sigma-Aldrich). Analysis of FFAs by gas chromatography-mass spectrometry-based (GC-MSbased) profiling is described in the Supplemental Methods.

Analysis of glucose metabolism. After overnight fasting, glucose levels in the mice were determined using a standard glucose meter (Contour, Bayer). The animals were then injected with glucose (i.p., $2 \mathrm{~g} / \mathrm{kg}$ body weight), and plasma glucose levels were determined at the indicated time points. Plasma insulin levels were determined with Ultra Sensitive Mouse Insulin ELISA (Crystal Chem).

Adipose tissue explants. Gonadal fat pads were removed from animals and washed in PBS. The fat pads were weighed, minced, and incubated in M199 medium (Gibco; Thermo Fisher Scientific) supplemented with $10 \%$ fetal bovine serum and $1 \%$ penicillin-streptomycin at $37^{\circ} \mathrm{C}$ and $5 \% \mathrm{CO}_{2}$. After 2 hours, fresh medium was added and the explants incubated overnight. Next, the explants were treated with M199 medium supplemented with 1\% fatty acid-free BSA, 1\% penicillin-streptomycin, and the indicated substances $(500 \mu \mathrm{M}$ IBMX, 5 $\mu \mathrm{g} / \mathrm{ml}$ insulin, $10 \mu \mathrm{M}$ forskolin, $100 \mathrm{nM} \mathrm{L} \mathrm{[-]} \mathrm{NE,} 10 \mu \mathrm{M}[-] \mathrm{IPT})$ for 72 hours (chronic) or 60 minutes (acute). The amounts of intracellular and released FFAs or released glycerol were quantified using commercial kits. The determination of lipases and esterase activities is detailed in the Supplemental Methods.

Analysis of human adipose tissue for SORLA expression. RNA from visceral adipose tissue was extracted using RNeasy Lipid Tissue Mini Kit (QIAGEN). Quantity and integrity of RNA was monitored with NanoVue plus Spectrophotometer (GE Healthcare). $1 \mu \mathrm{g}$ total RNA was reverse transcribed with standard reagents (Life Technologies) and cDNA processed for TaqMan probe-based q-PCR using the QuantStudio 6 Flex Real-Time PCR System (Life Technologies). Expression of SORL1 (Hs00268342_m1), Ucp1 (Mm01244861_m1), Cidea (Mm00432554_m1), Elovl3 (Mm00468164_m1), and BmpR1b (Mm03023971_m1) were calculated by standard curve method and normalized to the expression of 18S rRNA (4352930E) or Gapdh (Mm99999915_g1) (Life Technologies). 
For quantification of SORLA protein levels, we used a custommade ELISA described previously (39). In brief, wells were coated with goat anti-SORLA IgG in coating buffer $(100 \mathrm{mM}$ sodium bicarbonate, $\mathrm{pH} 9.8$ ) at $4^{\circ} \mathrm{C}$ overnight. After blocking with $2.5 \%$ casein (SigmaAldrich), human WAT protein lysates were loaded and incubated overnight at $4^{\circ} \mathrm{C}$. The next day, the plates were washed and incubated with rabbit anti-SORLA antibody (IgG 5387) and thereafter with horseradish peroxidase-conjugated anti-rabbit IgG. Colorimetric staining was performed by adding o-Phenylenediamine (Pierce) and measured on a microplate reader (BioTek) at $450 \mathrm{~nm}$.

Statistics. Statistical analyses were performed using GraphPad Prism 6 software. Data are presented as mean \pm SEM. For comparing 2 groups, unpaired 2-tailed Student's $t$ test was used. For experiments with more than 2 groups, unmatched 1-way ANOVA with Tukey's multiple comparisons test was used. When the same samples or animals received multiple treatments, then repeated-measures 2-tailed 1-way ANOVA, followed by Tukey's multiple comparisons test, was used. For experiments with more than 2 factors, repeated-measures 2-way ANOVA with Šidák multiple comparisons test was applied. $P$ values of less than 0.05 were considered significant. The Pearson's correlation coefficient was calculated with ImageJ software (NIH) to test for correlation between 2 parameters. Densitometric scanning of Western blot signals was also done with Image J software.

Study approval. The study on human adipose specimens was approved by the Ethics Committee of the University of Leipzig (approval no. 159-12-21052012) and performed in accordance with the declaration of Helsinki. All subjects gave written, informed con- sent before taking part in this study. All experiments involving mice were conducted following approval by local authorities of the Federal State of Berlin (X9012/12; G0339/12).

\section{Author contributions}

VS, NS, SK, XY, and GO designed and performed experiments. $\mathrm{MB}$ and MK provided essential human biospecimens. VS, AS, MK, MNP, and TEW evaluated data. TEW wrote the manuscript.

\section{Acknowledgments}

We are indebted to Aygul Subkhangulova for helpful discussions, to Tobias Opialla for providing the fatty acid mass spectral library, and to Arnd Heusser for help with the NMR analysis. C. Kruse, M. Liekweg, K. Kampf, S. Nilsson, M. Schmeisser, D. Kern, and J. Diesbach provided expert technical assistance. Studies were funded by the Deutsche Forschungsgemeinschaft (Wi1158/11-1) and the European Research Council (to T.E. Willnow), the Helmholtz Association (International Research Group Program and iCEMED) (to V. Schmidt), the Bundesministerium für Bildung und Forschung (DZD grant 01GI0922) (to A. Schürmann), and the Swedish Research Council (no. 12203 to G. Olivecrona).

Address correspondence to: Thomas E. Willnow or Vanessa Schmidt, Max-Delbrueck-Center for Molecular Medicine, Robert-Roessle-Str. 10, D-13125 Berlin, Germany. Phone: 49.30.9406.2569; E-mail: willnow@mdc-berlin.de (T.E. Willnow). Phone: 49.30.9406.3412; E-mail: vanessa.schmidt@mdcberlin.de (V. Schmidt).
1. Thorleifsson G, et al. Genome-wide association yields new sequence variants at seven loci that associate with measures of obesity. Nat Genet. 2009; 41(1):18-24.

2. Smith EN, et al. Longitudinal genome-wide association of cardiovascular disease risk factors in the Bogalusa heart study. PLoS Genet. 2010;6(9):e1001094.

3. Meyre D, et al. Genome-wide association study for early-onset and morbid adult obesity identifies three new risk loci in European populations. Nat Genet. 2009;41(2):157-159.

4. Willer CJ, et al. Six new loci associated with body mass index highlight a neuronal influence on body weight regulation. Nat Genet. 2009;41(1):25-34.

5. Willnow TE, Petersen CM, Nykjaer A. VPS1OPdomain receptors - regulators of neuronal viability and function. Nat Rev Neurosci. 2008;9(12):899-909.

6. Andersen OM, et al. Neuronal sorting proteinrelated receptor sorLA/LR11 regulates processing of the amyloid precursor protein. Proc Natl Acad Sci U S A. 2005;102(38):13461-13466.

7. Rohe M, et al. Sortilin-related receptor with A-type repeats (SORLA) affects the amyloid precursor protein-dependent stimulation of ERK signaling and adult neurogenesis. J Biol Chem. 2008;283(21):14826-14834.

8. Lambert JC, et al. Meta-analysis of 74,046 individuals identifies 11 new susceptibility loci for Alzheimer's disease. Nat Genet.
2013;45(12):1452-1458.

9. Parks BW, et al. Genetic control of obesity and gut microbiota composition in response to high-fat, high-sucrose diet in mice. Cell Metab. 2013;17(1):141-152.

10. Klinger SC, et al. SorLA regulates the activity of lipoprotein lipase by intracellular trafficking. J Cell Sci. 2011;124(pt 7):1095-1105.

11. Caglayan S, et al. Lysosomal sorting of amyloidbeta by the SORLA receptor is impaired by a familial Alzheimer's disease mutation. Sci Transl Med. 2014;6(223):223ra20.

12. Schmidt V, et al. SorLA/LR11 regulates processing of amyloid precursor protein via interaction with adaptors GGA and PACS-1.J Biol Chem. 2007;282(45):32956-32964.

13. Zechner R, et al. FAT SIGNALS - lipases and lipolysis in lipid metabolism and signaling. Cell Metab. 2012;15(3):279-291.

14. Lafontan M, Langin D. Lipolysis and lipid mobilization in human adipose tissue. Prog Lipid Res. 2009;48(5):275-297.

15. Stralfors P, Bjorgell P, Belfrage P. Hormonal regulation of hormone-sensitive lipase in intact adipocytes: identification of phosphorylated sites and effects on the phosphorylation by lipolytic hormones and insulin. Proc Natl Acad Sci US A. 1984;81(11):3317-3321.

16. Belfrage P, Fredrikson G, Nilsson NO, Stralfors P. Regulation of adipose-tissue lipolysis by phosphorylation of hormone-sensitive lipase. Int J Obes. 1981;5(6):635-641.
17. Nielsen TS, Jessen N, Jorgensen JO, Moller N, Lund S. Dissecting adipose tissue lipolysis: molecular regulation and implications for metabolic disease. JMol Endocrinol. 2014;52(3):R199-R222.

18. Whittle AJ, et al. Soluble LR11/SorLA represses thermogenesis in adipose tissue and correlates with BMI in humans. Nat Commun. 2015;6:8951.

19. Vijayakumar A, et al. Targeted loss of GHR signaling in mouse skeletal muscle protects against high-fat diet-induced metabolic deterioration. Diabetes. 2012;61(1):94-103.

20. Blouet C, Liu SM, Jo YH, Chua S, Schwartz GJ. TXNIP in Agrp neurons regulates adiposity, energy expenditure, and central leptin sensitivity. J Neurosci. 2012;32(29):9870-9877.

21. Liu P, Ying Y, Zhao Y, Mundy DI, Zhu M, Anderson RG. Chinese hamster ovary K2 cell lipid droplets appear to be metabolic organelles involved in membrane traffic. J Biol Chem. 2004;279(5):3787-3792.

22. Ronnett GV, Knutson VP, Lane MD. Insulininduced down-regulation of insulin receptors in 3T3-L1 adipocytes. Altered rate of receptor inactivation. J Biol Chem. 1982;257(8):4285-4291.

23. Marshall S. Kinetics of insulin receptor internalization and recycling in adipocytes. Shunting of receptors to a degradative pathway by inhibitors of recycling. J Biol Chem. 1985;260(7):4136-4144.

24. Burgert T, et al. SORLA-dependent and -independent functions for PACS1 in control of amyloidogenic processes. Mol Cell Biol. 2013;33(21):4308-4320.

25. Fjorback AW, et al. Retromer Binds the FANSHY 
Sorting Motif in SorLA to Regulate Amyloid Precursor Protein Sorting and Processing. J Neurosci. 2012;32(4):1467-1480.

26. Pottier C, et al. High frequency of potentially pathogenic SORL1 mutations in autosomal dominant early-onset Alzheimer disease. Mol Psychiatry. 2012;17(9):875-879.

27. Jensen M, De Meyts P. Molecular mechanisms of differential intracellular signaling from the insulin receptor. Vitam Horm. 2009;80:51-75.

28. Jacobsen L, et al. Molecular characterization of a novel human hybrid-type receptor that binds the a2-macroglobulin receptor-associated protein. J Biol Chem. 1996;271(49):31379-31383.

29. Yamazaki H, et al. Elements of neural adhesion molecules and a yeast vacuolar protein sorting receptor are present in a novel mammalian low density lipoprotein receptor family member. JBiol Chem. 1996;271(40):24761-24768.
30. Taira K, et al. LR11, a mosaic LDL receptor family member, mediates the uptake of ApoE-rich lipoproteins in vitro. Arterioscler Thromb Vasc Biol. 2001;21(9):1501-1506.

31. Nilsson SK, Lookene A, Beckstead JA, Gliemann J, Ryan RO, Olivecrona G. Apolipoprotein A-V interaction with members of the low density lipoprotein receptor gene family. Biochemistry. 2007;46(12):3896-3904.

32. Wheatley KE, Nogueira LM, Perkins SN, Hursting SD. Differential effects of calorie restriction and exercise on the adipose transcriptome in dietinduced obese mice. JObes. 2011;2011:265417.

33. Choi YH, et al. Alterations in regulation of energy homeostasis in cyclic nucleotide phosphodiesterase 3B-null mice. J Clin Invest. 2006;116(12):3240-3251.

34. Pal A, et al. PTEN mutations as a cause of constitutive insulin sensitivity and obesity. $\mathrm{N} \mathrm{EnglJ}$
Med. 2012;367(11):1002-1011.

35. Garcia-Cao I, et al. Systemic elevation of PTEN induces a tumor-suppressive metabolic state. Cell. 2012;149(1):49-62.

36. Dodt C, Lonnroth P, Fehm HL, Elam M. The subcutaneous lipolytic response to regional neural stimulation is reduced in obese women. Diabetes. 2000;49(11):1875-1879.

37. Ryden M, Andersson DP, Bernard S, Spalding $\mathrm{K}$, Arner P. Adipocyte triglyceride turnover and lipolysis in lean and overweight subjects. J Lipid Res. 2013;54(10):2909-2913.

38. Langin D, et al. Adipocyte lipases and defect of lipolysis in human obesity. Diabetes. 2005;54(11):3190-3197.

39. Schmidt V, et al. Quantitative modelling of amyloidogenic processing and its influence by SORLA in Alzheimer's disease. EMBO J. 2012;31(1):187-200. 